\title{
A Limit Theorem for Turbulent Diffusion
}

\author{
H. Kesten ${ }^{1}$ and G. C. Papanicolaou ${ }^{2}$ \\ ${ }^{1}$ Cornell University, Ithaca, New York 14853, USA \\ ${ }^{2}$ Courant Institute, New York University, New York, New York 10012, USA
}

\begin{abstract}
We show under some specific conditions that the formal diffusion approximation for the motion of a particle in a random velocity field is valid.
\end{abstract}

\section{Introduction}

Let $V(x)$ be a random velocity field with given statistical characteristics. Let $x(t)$ be the particle trajectories in $\mathbb{R}^{d}$ satisfying

$$
\frac{d x(t)}{d t}=V(x(t)), \quad x(0)=x .
$$

The turbulent diffusion problem consists of analyzing the statistical properties of the trajectories $x(t)$ under various hypotheses on the random velocity field $V(x)$. In particular, in many theoretical investigations, [1-3], one wants to find conditions under which the particle trajectories have classical diffusive behaviour and to compute the diffusion and drift coefficients in terms of the statistical properties of the random velocity field $V$.

We shall consider here this problem in what is perhaps the simplest situation, namely when

$$
V(x)=v+\varepsilon F(x),
$$

where $v$ is a constant nonzero vector representing the mean velocity, $F(x)$ is a given zero-mean stationary random field and $\varepsilon>0$ is a small dimensionless parameter measuring the size of the fluctuations. More precise conditions are given in the next section. For $\varepsilon$ small and $t$ large $\left(t \sim \varepsilon^{-2}\right.$ when $v$ and $F$ are given independently of $\varepsilon) x(t)-v t$ will behave like a diffusion process. This is the content of Theorem 1 in the next section.

By using formal perturbation theory, as in [4] for example, we shall now derive formulas for the diffusion and drift coefficients of the limiting diffusion process. Again, in Theorem 1 we show that these formulas are indeed the correct ones. 


\section{Define}

$$
x^{\varepsilon}(t)=x\left(t / \varepsilon^{2}\right)-\frac{t}{\varepsilon^{2}} v,
$$

which satisfies the equation

$$
\begin{aligned}
\frac{\partial x^{\varepsilon}(t)}{\partial t} & =\frac{1}{\varepsilon} F\left(x^{\varepsilon}(t)+\frac{t}{\varepsilon^{2}} v\right), \quad t>0, \\
x^{\varepsilon}(0) & =x .
\end{aligned}
$$

We write $x^{\varepsilon}(t)=x^{\varepsilon}(t ; x)$ to indicate dependence on the initial point. We also write $x^{\varepsilon}(t ; s, x)$ when (1.4) is satisfied for $t>s$ with $x^{\varepsilon}(s ; s, x)=x$. Clearly $x\left(t / \varepsilon^{2}\right)-v t / \varepsilon^{2}$ $=x^{\varepsilon}(t ; 0, x)$. Let $f(x)$ be a smooth function on $\mathbb{R}^{d}$ and let

$$
u^{\varepsilon}(t, s, x)=f\left(x^{\varepsilon}(t ; s, x)\right) .
$$

From the relation

$$
x^{\varepsilon}(t, s, x)=x^{\varepsilon}\left(t, s+h, x^{\varepsilon}(s+h, s, x)\right)
$$

we see that this function satisfies the adjoint or backward Liouville equation

$$
\begin{aligned}
& \frac{\partial u^{\varepsilon}}{\partial s}+\frac{1}{\varepsilon} F\left(x+\frac{s}{\varepsilon^{2}} v\right) \cdot \frac{\partial u^{\varepsilon}}{\partial x}=0, \quad s<t, \\
& u^{\varepsilon}(t, t, x)=f(x) .
\end{aligned}
$$

Here $\partial / \partial x$ denotes the gradient operator and the dot stands for Euclidean inner product. In integral form (1.6) becomes

$$
u^{\varepsilon}(t, s, x)=f(x)+\frac{1}{\varepsilon} \int_{s}^{t} F\left(x+\frac{\sigma}{\varepsilon^{2}} v\right) \cdot \frac{\partial u^{\varepsilon}}{\partial x}(t, \sigma, x) d \sigma .
$$

We iterate (1.7) once and take expected values. Since $E\{F(x)\}=0$ we obtain

$$
\begin{aligned}
E\left\{u^{\varepsilon}(t, s, x)\right\} & =f(x) \\
& +\frac{1}{\varepsilon^{2}} \int_{s}^{t} \int_{\sigma}^{t} E\left\{F\left(x+\frac{\sigma}{\varepsilon^{2}} v\right) \cdot \frac{\partial}{\partial x}\left(F\left(x+\frac{\lambda}{\varepsilon^{2}} v\right) \cdot \frac{\partial u^{\varepsilon}(t, \lambda, x)}{\partial x}\right)\right\} d \lambda d \sigma .
\end{aligned}
$$

Now we take $u^{\varepsilon}$ outside the expectation sign on the right and average it as if it were independent of $F$. Justifying the equivalent of this step is what requires most of the work in the proof of Theorem 1. Retaining the equality sign as an approximate equality for $\varepsilon$ small we have

$$
\begin{aligned}
E\left\{u^{\varepsilon}(t, s, x)\right\}= & f(x) \\
+ & \frac{1}{\varepsilon^{2}} \int_{s}^{t} \int_{\sigma}^{t} E\left\{F\left(x+\frac{\sigma}{\varepsilon^{2}} v\right) \cdot \frac{\partial}{\partial x} F\left(x+\frac{\lambda}{\varepsilon^{2}} v\right) \cdot \frac{\partial}{\partial x}\right\} \\
& \cdot E\left\{u^{\varepsilon}(t, \lambda, x)\right\} d \lambda d \sigma,
\end{aligned}
$$


which in differential form becomes

$$
\begin{aligned}
-\frac{\partial}{\partial s} E\left\{u^{\varepsilon}(t, s, x)\right\}= & \frac{1}{\varepsilon^{2}} \int_{s}^{t} E\left\{F\left(x+\frac{s}{\varepsilon^{2}} v\right) \cdot \frac{\partial}{\partial x} F\left(x+\frac{\lambda}{\varepsilon^{2}} v\right) \cdot \frac{\partial}{\partial x}\right\} \\
& \cdot E\left\{u^{\varepsilon}(t, \lambda, x)\right\} d \lambda, \quad s<t, \\
E\left\{u^{\varepsilon}(t, t, x)\right\}= & f(x) .
\end{aligned}
$$

When the correlations of $F$ with itself at two different points decay rapidly with the distance between the points, (1.8) can be simplified to

$$
\begin{aligned}
-\frac{\partial}{\partial s} E\left\{u^{\varepsilon}(t, s, x)\right\}= & \int_{0}^{\infty} E\left\{F(x) \cdot \frac{\partial}{\partial x} F(x+\sigma v) \cdot \frac{\partial}{\partial x}\right\} \\
& \cdot E\{u(t, s, x)\} d \sigma, \quad s<t, \\
E\left\{u^{\varepsilon}(t, t, x)\right\}= & f(x) .
\end{aligned}
$$

Here we have used also the stationary of $F$ and have performed a change of variables (cf. [4]; this step is sometimes called the longtime Markovian approximation. The approximation that leads to (1.8) is called the smoothing approximation). Put

$$
\mathscr{L} g(x)=\int_{0}^{\infty} E\left\{F(x) \cdot \frac{\partial}{\partial x}\left(F(x+t v) \cdot \frac{\partial g(x)}{\partial x}\right)\right\} d t .
$$

This is a diffusion operator with constant coefficients:

$$
\mathscr{L} g(x)=\frac{1}{2} \sum_{i, j=1}^{d} a_{i j} \frac{\partial^{2} g(x)}{\partial x_{i} \partial x_{j}}+\sum_{j=1}^{d} b_{j} \frac{\partial g(x)}{\partial x_{j}},
$$

where

$$
a_{i j}=\int_{0}^{\infty} E\left\{F_{i}(x) F_{j}(x+t v)\right\} d t+\int_{0}^{\infty} E\left\{F_{i}(x+t v) F_{j}(x)\right\} d t
$$

and

$$
b_{j}=\int_{0}^{\infty} \sum_{i=1}^{d} E\left\{F_{i}(x) \frac{\partial F_{j}}{\partial x_{i}}(x+t v)\right\} d t .
$$

Let

$$
w^{\varepsilon}(t, s, x)=E\left\{u^{\varepsilon}(t, s, x)\right\}=E\left\{f\left(x^{\varepsilon}(t ; s, x)\right)\right\} .
$$

We have shown formally that as $\varepsilon \rightarrow 0 w^{\varepsilon}(t, s, x)$ tends to $w(t, s, x)$ which satisfies

$$
\begin{aligned}
-\frac{\partial w}{\partial s} & =\mathscr{L} w, \quad s<t, \\
w(t, t, x) & =f(x) .
\end{aligned}
$$

Since $\mathscr{L}$ has constant coefficients it follows that $w(t, s, x)$ depends on $t-s$ only. Setting $s=0$ we see that as $\varepsilon \rightarrow 0$

$$
E\left\{f\left(x\left(t / \varepsilon^{2}\right)-v t / \varepsilon^{2}\right)\right\} \rightarrow w(t, x),
$$


where $w(t, x)$ is the solution of the diffusion equation

$$
\frac{\partial w}{\partial t}=\mathscr{L} w, \quad t>0, \quad w(0, x)=f(x) .
$$

(1.15) and (1.16) show that $x\left(t / \varepsilon^{2}\right)-t v / \varepsilon^{2}$ converges to the diffusion process generated by $\mathscr{L}$ as $\varepsilon \downarrow 0$. This is exactly what is proved in Theorem 1 .

The method of analysis that is followed here works also for more general velocity fields $V(x)$ where the mean velocity is not a constant $v$ as in (1.2). One can, for example, obtain theorems that cover some cases treated in [1] concerning the turbulent dispersion of particles in the atmospheric boundary layer. Along different lines, one can also generalize Theorem 1 to discuss the motion of several particles in the same field. An example of this is given in Theorem 2.

\section{Statement of the Main Theorem}

We begin by introducing several hypotheses regarding the vector field $F(x)$.

Let $(\Omega, \mathscr{F}, P)$ be a probability space and let $F(x, \omega): \mathbb{R}^{d} \times \Omega \rightarrow \mathbb{R}^{d}$ be jointly measurable relative to $\mathscr{B} \times \mathscr{F}$, where $\mathscr{B}=\sigma$-algebra of Borel sets in $\mathbb{R}^{d}$. We assume that for $P$ almost all $\omega$ the random field $F(x)=\left(F_{i}(x, \omega)\right)$ is three times continuously differentiable in $x=\left(x_{1}, \ldots, x_{d}\right)$. We also assume that $F(x)$ is strictly stationary, i.e., for each $h \in \mathbb{R}^{d}$ and points $y_{1}, y_{2}, \ldots, y_{n}$ in $\mathbb{R}^{d}$ the joint distribution of

$$
F\left(y_{1}+h\right), F\left(y_{2}+h\right), \ldots, F\left(y_{n}+h\right)
$$

is the same as that of

$$
F\left(y_{1}\right), F\left(y_{2}\right), \ldots, F\left(y_{n}\right) \text {. }
$$

We define a process $x(t)=x(t, \omega)$ with values in $\mathbb{R}^{d}, t \geqq 0, \omega \in \Omega$, as the solution of the differential equation

$$
\frac{d x(t, \omega)}{d t}=v+\varepsilon F(x(t, \omega), \omega), \quad x(0, \omega)=x_{0} \in \mathbb{R}^{d} .
$$

Here $v \in \mathbb{R}^{d}, v \neq 0$, is a fixed vector, $\varepsilon \in(0,1]$ is a small parameter which we shall let tend to zero and $x_{0}$ is the (nonrandom) initial position. It will be part of our theorem that under the stated conditions plus (2.2) below, (2.1) has with probability one a unique solution for all $t \geqq 0$. We are interested in the asymptotic behavior of $x(t, \omega)$ as $\varepsilon \rightarrow 0$ and $t \rightarrow \infty$ with $\varepsilon^{2} t=$ constant as described in the introduction. For this purpose we need additional hypotheses on $F(x, \omega)$ as follows.

Let $\beta=\left(\beta_{1}, \ldots, \beta_{d}\right)$, with $\beta_{i} \geqq 0$ integers, be a multi-index, denote by $D^{\beta}$ partial derivatives as usual and set $|\beta|=\beta_{1}+\ldots+\beta_{d}$. We shall assume that for each $0 \leqq M<\infty$ and each $\beta$ with $0 \leqq|\beta| \leqq 3$

$$
\int_{\Omega}\left[\left.\sup _{|x| \leqq M}\left|D^{\beta} F(x, \omega)\right|\right|^{\max (8, d)} P(d \omega)=E\left\{\left[\sup _{|x| \leqq M}\left|D^{\beta} F(x)\right|\right]^{\max (8, d)}\right\}<\infty .\right.
$$

Since $F$ is stationary, if (2.2) holds for one $M$, say $M=1$, then it holds for all $M<\infty$. 
We assume that $F$ has mean zero, i.e.,

$$
\int_{\Omega} \mathrm{F}(\mathrm{x}, \omega) \mathrm{P}(\mathrm{d} \omega)=\mathrm{E}\{\mathrm{F}(\mathrm{x})\}=0 .
$$

Let $\Lambda \in \mathscr{B}\left(\mathbb{R}^{d}\right)$ and let $\mathscr{F}_{\Lambda}$ denote the minimal $\sigma$-algebra of subsets of $\Omega$ in $\mathscr{F}$ generated by sets of the form

$$
\{\omega \in \Omega \mid F(x, \omega) \in A\}, \quad A \in \mathscr{B}, \quad x \in \Lambda .
$$

With $v \in \mathbb{R}^{d}, v \neq 0$ fixed, and $0 \leqq M \leqq \infty$ we shall use the notation

$$
\mathscr{F}_{s}^{t}(v, M)=\mathscr{F}_{A}, \quad-\infty \leqq s \leqq t \leqq+\infty,
$$

where

$$
\Lambda=\left\{x \in R^{d}\left|s \leqq \frac{(x, v)}{|v|} \leqq t,\right| x-\frac{(x, v) v}{|v|^{2}} \mid \leqq M\right\} .
$$

Here (, ) denotes the usual Euclidean inner product of vectors. When $v \neq 0$ and $M$ are fixed we write $\mathscr{F}_{s}^{t}$ for short.

We shall assume that $P$ is strongly mixing on the $\sigma$-algebras $\mathscr{F}_{s}^{t}$ as follows. With $v \neq 0$ and $M<\infty$ fixed define

$$
\alpha(t, v, M)=\sup _{s} \sup _{\substack{A \in \mathscr{F} \\ B \in \mathscr{F} s_{-\infty}^{\infty}+t}}|P(A B)-P(A) P(B)|, \quad t \geqq 0 .
$$

If $\alpha(t)=\alpha(t, v, M) \rightarrow 0$ as $t \rightarrow \infty$, we say that $P$ is strongly mixing at the rate $\alpha(\cdot)$. For our result we assume that the mixing rate satisfies

$$
\int_{0}^{\infty}\{\alpha(t, v, M)\}^{1 / p} d t<\infty
$$

for $p=6+2 d$. Define $x^{\varepsilon}(t, \omega)$ by

$$
x^{\varepsilon}(t, \omega)=x\left(\frac{t}{\varepsilon^{2}}, \omega\right)-\frac{t}{\varepsilon^{2}} v,
$$

where $x(t, \omega)$ is the solution of (2.1). Let $Q^{\varepsilon}$ denote the probability measure induced by $x^{\varepsilon}(t)$ on $C=C\left([0, \infty) ; \mathbb{R}^{d}\right)$, the space of continuous functions from $[0, \infty)$ to $\mathbb{R}^{d}$.

Theorem 1. If $v \neq 0$ and the above assumptions hold (in particular (2.2), (2.3), and (2.7)) then (2.1) has with probability one a unique solution for all $t \geqq 0$. Moreover $Q^{\varepsilon}$ converges weakly on $C$ as $\varepsilon \downarrow 0$ to the measure $Q$ corresponding to the diffusion process in $\mathbb{R}^{d}$ with infinitesimal generator $\mathscr{L}$ given by (1.11), (1.12) and with initial position $x_{0}$.

Remark 1. For the definition and general theory of weak convergence the reader is referred to [5]. For the case of the infinite time interval $[0, \infty)$, as here, $[6]$ is the most helpful.

Since the coefficients of $\mathscr{L}$ in (1.12) are independent of $x$, the measure $Q$ is essentially the Brownian motion measure. 
Let

$$
r_{i j}(y)=E\left\{F_{i}(x) F_{j}(x+y)\right\}, \quad i, j=1, \ldots, d,
$$

be the correlation matrix of the fluctuations of the velocity field. It is clear from (2.2) that $r_{i j, i}=\frac{\partial}{\partial y_{i}} r_{i j}$ is well defined. Moreover, by our mixing condition (2.7) and Theorem 17.2.2 of [7] $r_{i j}(y)=r_{j i}(-y)$ and $r_{i j, i}(y)$ decay rapidly as $|y| \rightarrow \infty$ and the coefficients $a_{i j}$ and $b_{j}$ in (1.12) may be represented by the following absolutely convergent integrals:

$$
a_{i j}=\int_{-\infty}^{+\infty} r_{i j}(t v) d t, \quad b_{j}=\int_{0}^{\infty} \sum_{i=1}^{d} r_{i j, i}(t v) d t .
$$

One can use Theorem 1 to obtain the asymptotic form of the probability measure of two (or more) trajectories of (2.1) starting from different initial points. The correct form of the generator of the limiting diffusion is again obtained easily by formal perturbation theory as in the introduction. Let $x(t, \omega)$ be as in $(2.1)$ and let $y(t, \omega)$ be the solution of $(2.1)$ with $y(0, \omega)=y_{0} \in \mathbb{R}^{d}$, with $y_{0} \neq x_{0}$. Let $x^{\varepsilon}(t, \omega)$ be defined by $(2.8)$ and set $y^{\varepsilon}(t, \omega)=y\left(\frac{t}{\varepsilon^{2}}, \omega\right)-\frac{t}{\varepsilon^{2}} v$. Let $\hat{Q}^{\varepsilon}$ be the probability measure on $C\left([0, \infty) ; \mathbb{R}^{2 d}\right)$ induced by $\left(x^{\varepsilon}(t), y^{\varepsilon}(t)\right)$.

Theorem 2. Under the same hypotheses as in Theorem $1 x^{\varepsilon}(t, \omega)$ and $y^{\varepsilon}(t, \omega)$ are with probability one uniquely defined for all $t \geqq 0$. Moreover $\hat{Q}^{\varepsilon}$ converges weakly as $\varepsilon \downarrow 0$ to the measure $\hat{Q}$ corresponding to the diffusion process in $\mathbb{R}^{2 d}$ with initial position $x_{0}$, $y_{0}$ and with infinitesimal generator

$$
\hat{\mathscr{L}}=\mathscr{L}_{x}+\mathscr{L}_{y}+\mathscr{L}_{x y},
$$

where $\mathscr{L}_{x}$ is the operator (1.11), $\mathscr{L}_{y}$ is the same as (1.11) with $x$ derivatives replaced by $y$ derivatives and $\mathscr{L}_{x y}$ is given by

$$
\mathscr{L}_{x y}=\sum_{i, j=1}^{d} \int_{-\infty}^{\infty} r_{i j}(y-x+t v) d t \frac{\partial^{2}}{\partial x_{i} \partial y_{i}} .
$$

Remark 2. Note that the generator $\hat{\mathscr{L}}$ does not have constant coefficients because of the cross-derivative generator $\mathscr{L}_{x y}$.

Theorem 1 (and implicitly Theorem 2 ) will be proven in the next section. In the Appendix we construct some examples of random fields which satisfy the hypotheses of Theorem 1. In particular, Theorem 1 applies to Gaussian random fields with certain rational spectral densities.

\section{Proof of Theorem 1}

Observe that

$$
\frac{d}{d t}\left(x\left(\frac{t}{\varepsilon^{2}}\right), v\right)=\frac{1}{\varepsilon^{2}}|v|^{2}+\frac{1}{\varepsilon}\left(F\left(x\left(\frac{t}{\varepsilon^{2}}\right)\right), v\right),
$$


which will usually be positive for small $\varepsilon$. This means that the $v$-components of $x\left(\frac{t_{1}}{\varepsilon^{2}}\right)$ and $x\left(\frac{t_{2}}{\varepsilon^{2}}\right)$ will usually differ at least by $\left(2 \varepsilon^{2}\right)^{-1}\left(t_{2}-t_{1}\right)$ and consequently $F\left(x\left(\frac{t_{1}}{\varepsilon^{2}}\right)\right)$ and $F\left(x\left(\frac{t_{2}}{\varepsilon^{2}}\right)\right)$ will be almost independent by virtue of our mixing assumption. Even though this argument does not appear explicitly in our proof it has its reflection in the crucial Condition III of Theorem 3 below.

Our first step will be to reduce Theorem 1 to a theorem of a more familiar form, in which the random field also depends on time, but in such a manner that two values corresponding to widely separated times are almost independent.

Step (i). Define

$$
G(t, x, \varepsilon, \omega)=F(x+t v, \omega),
$$

and let $x^{\varepsilon}(t)$ be as in (2.8). (In our case $G$ does not depend on $\varepsilon$, but the general Theorem 3 below permits such dependence.) It is immediate that

$$
\frac{d x^{\varepsilon}(t)}{d t}=\frac{1}{\varepsilon} G\left(\frac{t}{\varepsilon^{2}}, x^{\varepsilon}(t), \varepsilon\right), \quad x^{\varepsilon}(0)=x_{0} .
$$

We next verify that $G$ satisfies the Conditions I-VI below.

I. $G$ is jointly measurable in all its arguments and a.s. in $C^{3}\left(\mathbb{R}^{d}\right)$ as a function of $x$ for each $t, \varepsilon$.

II. The process $\{G(t, \cdot, \varepsilon, \omega)\}_{t \geqq 0}$ is stationary in $t$ for each fixed $\varepsilon$.

III. Let

$$
\mathscr{G}_{s}^{t}(\varepsilon, M)=\sigma\{G(u, x, \varepsilon, \cdot)|s \leqq u \leqq t,| x \mid \leqq M\}
$$

and

$$
\beta(t, M)=\sup _{\substack{s \geqq 0 \\ 0<\varepsilon \leqq 1}} \sup _{\substack{A \in \mathscr{C}_{0}^{s}(\varepsilon, M) \\ B \in \mathscr{G}_{s}^{0}+t(\varepsilon, M)}}|P(A B)-P(A) P(B)| .
$$

Then

$$
\int_{0}^{\infty}\{\beta(t, M)\}^{1 / p} d t<\infty
$$

for $p=6+2 d$.

IV. Write

$$
\bar{G}(x, \varepsilon)=E\{G(t, x, \varepsilon)\} .
$$

There exists a fixed $w \in \mathbb{R}^{d}$ such that for each $M<\infty$

$$
\lim _{\varepsilon \downarrow 0} \sup _{|x| \leqq M}\left|\frac{1}{\varepsilon} \bar{G}(x, \varepsilon)-w\right|=0 .
$$

$\mathrm{V}$. For each $M<\infty$ there exist a constant $C=C(M)$ independent of $t, z$ and $\varepsilon$ such that

$$
E\left\{\sup _{|x-z| \leqq M}\left|D^{\beta} G(t, x, \varepsilon)\right|^{\max (8, d)}\right\} \leqq C, \quad 0 \leqq|\beta| \leqq 3,
$$


and these integrals converge uniformly in $z$. [The derivatives in (3.7) are all $x$ derivatives.]

VI. The following limits exist uniformly on compact sets and are bounded functions of $x$ :

$$
\begin{aligned}
& A_{i j}(x)=\lim _{\varepsilon \downarrow 0} \int_{0}^{\infty} E\left\{G_{i}(0, x, \varepsilon) \tilde{G}_{j}(t, x, \varepsilon)\right\} d t, \\
& c_{i j}(x)=\lim _{\varepsilon \downarrow 0} \int_{0}^{\infty} E\left\{G_{i}(0, x, \varepsilon) \frac{\partial}{\partial x_{i}} \tilde{G}_{j}(t, x, \varepsilon)\right\} d t,
\end{aligned}
$$

where

$$
\tilde{G}=G-\bar{G} .
$$

[The integrals (3.8) and (3.9) converge absolutely on account of III, for the reasons given before (2.10).] In addition either the matrix $a_{i j}=\left(A_{i j}+A_{j i}\right)$ is twice continuously differentiable in $x$ and the vector

$$
b_{j}(x)=\sum_{i=1}^{d} c_{i j}(x)+w_{j}
$$

is uniformly Lipschitz continuous in $x$, or $A$ is strictly positive definite and continuous at each point $x \in \mathbb{R}^{d}$.

I, II, IV (with $w=0$ ), and V are immediate for the choice (3.1) of $G$ by our assumptions on $F$. To prove III for the choice (3.1) of $G$ note that for $|x| \leqq M$, $s \leqq u \leqq t$,

$$
s|v|-M \leqq|v|^{-1}(x+u v, v) \leqq t|v|+M, \quad\left|x+u v-\frac{(x+u v, v) v \mid}{|v|^{2}}\right| \leqq M .
$$

Consequently

$$
\mathscr{G}_{s}^{t}(\varepsilon, M) \subset \mathscr{F}^{t} \begin{gathered}
t|v|+M \\
s|v|-M
\end{gathered}(v, M)
$$

and

$$
\left.\beta(t, M) \leqq \alpha(t|v|-2 M)^{+}, v, M\right) .
$$

Thus (3.4) is immediate from (2.7). Finally the convergence and continuity properties of (3.8) and (3.9) are trivial in our case since the integrals are independent of $\varepsilon$ [by (3.1)] and of $x$ (by the stationarity of $F$ ). Indeed we have the explicit expressions

$$
\begin{aligned}
& a_{i j}(x)=\int_{0}^{\infty} E\left\{F_{i}(x) F_{j}(x+t v)+F_{i}(x+t v) F_{j}(v)\right\} d t, \\
& c_{i j}(x)=\int_{0}^{\infty} E\left\{F_{i}(x) \frac{\partial}{\partial x_{i}} F_{j}(x+t v)\right\} d t,
\end{aligned}
$$

exactly as in (1.12). 
We note that $a_{i j}=A_{i j}+A_{j i}$ is always nonnegative definite since it equals

$$
a_{i j}(x)=\lim _{\varepsilon \downarrow 0} \lim _{T \rightarrow \infty} \frac{1}{2 T} \int_{-T}^{+T} d s \int_{-T}^{+T} d t E\left\{\tilde{G}_{i}(s, x, \varepsilon) \tilde{G}_{j}(t, x, \varepsilon)\right\} .
$$

We also observe that even integrals of the form

$$
\int_{0}^{\infty} E\left\{F_{i}(x) F_{j}(y+t v)\right\} d t
$$

(which appear in $\mathscr{L}_{x y}$ in Theorem 2) are twice continuously differentiable by virtue of (2.2) and the rapid decrease of $r_{i j}(y)$ and its derivatives, which follows from (2.7) and Theorem 17.2.2 of [7] (see Remark 1).

Before stating the next theorem we comment that we have not made any continuity assumptions for $G$ as a function of $t$. Accordingly by a solution of (3.2) we mean from now on any continuous function $x^{\varepsilon}(\cdot)$ which satisfies

$$
x^{\varepsilon}(t, \omega)=x_{0}+\frac{1}{\varepsilon} \int_{0}^{t} G\left(\frac{\sigma}{\varepsilon^{2}}, x^{\varepsilon}(\sigma, \omega), \varepsilon, \omega\right) d \sigma, \quad t \geqq 0 .
$$

Theorem 3. Let $G$ be a random field which satisfies conditions $I-V I$. Then with probability one (3.13) has a unique solution for all $t \geqq 0$. Let $R^{\varepsilon}$ be the measure on $C\left([0, \infty) ; \mathbb{R}^{d}\right)$ induced by this solution. For each $f \in C^{2}\left(\mathbb{R}^{d}\right)$ put

$$
L f(x)=\frac{1}{2} \sum_{i, j=1}^{d} a_{i j}(x) \frac{\partial^{2} f(x)}{\partial x_{i} \partial x_{j}}+\sum_{j=1}^{d} b_{j} \frac{\partial f(x)}{\partial x_{j}} .
$$

Then $R^{\varepsilon}$ converges weakly to the probability measure $R$ on $C\left([0, \infty), \mathbb{R}^{d}\right)$ which corresponds to the diffusion with the infinitesimal generator $L$ and starting point $x_{0}$ (i.e., $R\left\{X(0)=x_{0}\right\}=1$ ).

It is clear from the above that Theorem 1 is indeed a special case of Theorem 3. Results like Theorem 3 were first proven by Khasminskii [8], in [9], and more recently by Borodin [10]. In fact Theorem 3 is very similar to Borodin's result. However, our proof differs from Borodin's in some important details. We circumvent the usual technique of breaking up the increments of $x^{\varepsilon}(t)$ into blocks and we end up with a weaker mixing condition than Borodin's (but our differentiability condition is stronger than Borodin's). Moreover, by introducing a truncation in Step (ii) we can get away with requiring III and V for each finite $M$ only, with the constants dependent on $M$. We actually exploit this in our explicit examples (see Appendix) which do not satisfy Borodin's conditions as they stand. Despite its length we shall therefore give a complete proof of Theorem 3 in Steps (ii)-(iv) below. After introducing the truncated process $x^{\varepsilon, M}$ in Step (ii) we show in Step (iii) that the corresponding family $Q^{\varepsilon, M}$ of measures is tight on $D\left([0, \infty), \mathbb{R}^{d}\right)$ (see $[5,6]$ for definition of $D)$. This is done by means of a mixing lemma proved in Step (iv). The Steps (v) and (vi) show that the measures $Q^{\varepsilon}$ actually have a limit as $\varepsilon \rightarrow 0$ and identify the limit. The truncation is not removed until the very last step.

Remark 3. In Theorem 3 we do not try to find the weakest possible differentiability condition in V. If $G$ is of the form $G^{(1)}+\varepsilon G^{(2)}$ then one can separately estimate the 
terms involving $G^{(2)}$ in (3.44) below. In this case it suffices that $G^{(1)}$ satisfies (3.7) for $|\beta| \leqq 3$ and $G^{(2)}$ satisfies (3.7) for $|\beta| \leqq 1$ only.

Step (ii). Let $\varphi_{M}(x)$ be a $C^{\infty}\left(\mathbb{R}^{d}\right)$ function such that $0 \leqq \varphi_{M} \leqq 1$,

$$
\varphi_{M}(x)=\left\{\begin{array}{lll}
1 & \text { if } & |x| \leqq \frac{M}{2} \\
0 & \text { if } & |x| \geqq M
\end{array}\right.
$$

and such that gradient $\left(\varphi^{M}(x)\right)$ is bounded uniformly in $x$, and $M \geqq 1$. We define the truncated field $G^{M}(\cdot)$ by

$$
G^{M}(t, x, \varepsilon, \omega)=G(t, x, \varepsilon, \omega) \varphi_{M}(x)
$$

and the corresponding process $x^{\varepsilon, M}(t, \omega)$ as the solution of

$$
x^{\varepsilon, M}(t, \omega)=x_{0}+\frac{1}{\varepsilon} \int_{0}^{t} G^{M}\left(\frac{\sigma}{\varepsilon^{2}}, x^{\varepsilon, M}(\sigma, \omega), \varepsilon, \omega\right) d \sigma, \quad t \geqq 0 .
$$

We assume $M>\left|x_{0}\right|$ throughout. At this stage we first prove that (3.16) and (3.13) have unique solutions as stated. First note that for each fixed $M$ and $T$

$$
\begin{aligned}
E & \left\{\frac{1}{\varepsilon} \int_{0}^{T}\left[\sup _{x}\left|G^{M}\left(\frac{t}{\varepsilon^{2}}, x, \varepsilon\right)\right|+\sup _{x}\left|\frac{\partial}{\partial x} G^{M}\left(\frac{t}{\varepsilon^{2}}, x, \varepsilon\right)\right|\right] d t\right\} \\
& \leqq \frac{C}{\varepsilon} \int_{0}^{T} E\left\{\left[\sup _{|x| \leqq M}\left|G\left(\frac{t}{\varepsilon^{2}}, x, \varepsilon\right)\right|+\sup _{|x| \leqq M}\left|\frac{\partial}{\partial x} G\left(\frac{t}{\varepsilon^{2}}, x, \varepsilon\right)\right|\right]\right\} d t<\infty
\end{aligned}
$$

so that almost surely

$$
\frac{1}{\varepsilon} \int_{0}^{T} \sup _{x}\left|G^{M}\left(\frac{t}{\varepsilon^{2}}, x, \varepsilon\right)\right| d t<\infty
$$

and

$$
\frac{1}{\varepsilon} \int_{0}^{T} \sup _{x}\left|\frac{\partial}{\partial x} G^{M}\left(\frac{t}{\varepsilon^{2}}, x, \varepsilon\right)\right| d t<\infty .
$$

For any $\omega$ for which this is satisfied there exists a $\delta>0$ such that

$$
\frac{1}{\varepsilon} \int_{s}^{s+\delta} \sup _{x}\left|\frac{\partial}{\partial x} G^{M}\left(\frac{t}{\varepsilon^{2}}, x, \varepsilon\right)\right| d t \leqq \frac{1}{2} \quad \text { for all } \quad 0 \leqq s \leqq s+\delta \leqq T .
$$

It follows from (3.17) that for each fixed $z \in \mathbb{R}^{d}, 0 \leqq s \leqq T-\delta$, the map

$$
f(s+u) \rightarrow z+\frac{1}{\varepsilon} \int_{s}^{s+u} G^{M}\left(\frac{t}{\varepsilon^{2}}, f(t), \varepsilon\right) d t, \quad 0 \leqq u \leqq \delta
$$

is a contraction on $C\left([s, s+\delta] ; \mathbb{R}^{d}\right)$ (with the sup norm) and therefore for each such $z, s$ there is a unique solution to

$$
f(s+u)=z+\frac{1}{\varepsilon} \int_{s}^{s+u} G^{M}\left(\frac{t}{\varepsilon^{2}}, f(t), \varepsilon\right) d t, \quad 0 \leqq u \leqq \delta .
$$


By iteration we see that (3.17) implies that almost surely (3.16) has a unique solution on $[0, T]$. This holds for all $M, T$, hence (3.16) has almost surely for each $M$ a unique solution for all $t \geqq 0$. Since $G^{M}(t, x, \varepsilon)=G(t, x, \varepsilon)$ for $|x| \leqq \frac{M}{2}$ we see that any solution $x^{\varepsilon, M}(\cdot)$ of (3.16) which satisfies $\left|x^{\varepsilon, M}(t)\right| \leqq \frac{M}{2}$ on $[0, T]$ is also a solution of $(3.13)$ in $[0, T]$. Now the solution of (3.16) satisfies

$$
\sup _{0 \leqq t \leqq T}\left|x^{\varepsilon, M}(t)\right| \leqq\left|x_{0}\right|+\frac{1}{\varepsilon} \int_{0}^{T} \sup _{|x|}\left|G^{M}\left(\frac{t}{\varepsilon^{2}}, x, \varepsilon\right)\right| d t
$$

so that existence of a solution of (3.13) will follow if we show

$$
\lim _{M \rightarrow \infty} P\left\{\frac{1}{\varepsilon} \int_{0}^{T} \sup _{|x|}\left|G^{M}\left(\frac{t}{\varepsilon^{2}}, x, \varepsilon\right)\right| d t>\frac{M}{4}\right\}=0 .
$$

However, (3.18) is easy because for any $\eta>0$

$$
\begin{aligned}
E & \left\{\frac{1}{\varepsilon} \int_{0}^{T} \sup _{x}\left|G^{M}\left(\frac{t}{\varepsilon^{2}}, x, \varepsilon\right)\right| d t\right\} \leqq \frac{\eta}{\varepsilon} M T \\
& +\frac{1}{\varepsilon} \int_{0}^{T} \sum_{n} E\left\{\sup _{\left|x-z_{n}\right| \leqq 1}\left|G\left(\frac{t}{\varepsilon^{2}}, x, \varepsilon\right)\right| I\left[\left|G\left(\frac{t}{\varepsilon^{2}}, x, \varepsilon\right)\right| \geqq \eta M\right]\right\} d t,
\end{aligned}
$$

where the sum is over a finite number of points $z_{n}$ such that the union of the balls $\left\{x:\left|x-z_{n}\right| \leqq 1\right\}$ covers $\{x:|x| \leqq M\}$. We can do this with at most $C_{1} M^{d}$ balls. The $n$th expectation in the sum is at most

$$
\begin{aligned}
& (\eta M)^{-d+1} E\left\{\sup _{\left|x-z_{n}\right| \leqq 1}\left|G\left(\frac{t}{\varepsilon^{2}}, x, \varepsilon\right)\right|^{d} \cdot I\left[\left|G\left(\frac{t}{\varepsilon^{2}}, x, \varepsilon\right)\right| \geqq \eta M\right]\right\} \\
& =o\left(M^{-d+1}\right), \quad M \rightarrow \infty .
\end{aligned}
$$

(by V). It follows that

$$
E\left\{\frac{1}{\varepsilon} \int_{0}^{T} \sup _{|x|}\left|G^{M}\left(\frac{t}{\varepsilon^{2}}, x, \varepsilon\right)\right| d t\right\}=o(M), \quad M \rightarrow \infty,
$$

which immediately implies (3.18).

Now that the existence of a solution to (3.13) has been established, the uniqueness of the solution again follows by the standard argument, given just below (3.17).

We return to the solution $x^{\varepsilon, M}$ of (3.16). Since $G^{M}(t, x, \varepsilon)=0$ for $|x|>M$ we have

$$
\left|x^{\varepsilon, M}(t, \omega)\right| \leqq M \quad \text { for all } t \geqq 0 .
$$

Until the end of Step (v), $\left|x_{0}\right|<M<\infty$ will remain fixed and we only deal with $x^{\varepsilon, M}$, $G^{M}$. For brevity we suppress the $M$ in the notation in Steps (iii) and (iv). The only property used in these steps which hinges on the truncation is (3.19).

Step (iii). Let $Q^{\varepsilon, M}$ be the measure induced by $x^{\epsilon, M}$ on $D\left([0, \infty) ; \mathbb{R}^{d}\right)$. In this step we show that for each fixed $M$ the family $\left\{Q^{\varepsilon, M}\right\}_{\varepsilon>0}$ is tight. By a result of Chentsov (see [5, p. 125ff.], especially the proofs of Theorems 15.4 and 15.6) it 
suffices for this to show

$$
E\left|x^{\varepsilon}(u)-x^{\varepsilon}(t)\right|^{r_{1}} \leqq C(u-t)^{r_{3}}
$$

and

$$
E\left|x^{\varepsilon}(s)-x^{\varepsilon}(t)\right|^{r_{1}}\left|x^{\varepsilon}(u)-x^{\varepsilon}(t)\right|^{r_{2}} \leqq C(u-s)^{1+r_{4}}
$$

for some $r_{1}, \ldots, r_{4}>0$ and $0 \leqq s \leqq t \leqq u \leqq T$ and $C=C(T)<\infty$ independent of $\varepsilon \in(0,1]$ and $s, t, u$. In turn (3.20) and (3.21) follow if we show that for $0 \leqq t \leqq u \leqq T$

$$
E\left\{\left|x^{\varepsilon}(u)-x^{\varepsilon}(t)\right|^{2} \Phi\right\} \leqq C_{1}(u-t) E^{1 / 8}\left\{\Phi^{8}\right\}
$$

for

$$
\Phi=\Phi(s, t)=\left|x^{\varepsilon}(t)-x^{\varepsilon}(s)\right|^{r}, \quad 0 \leqq s \leqq t \quad \text { and } \quad 0 \leqq r \leqq 2
$$

and $C_{1}=C_{1}(T)<\infty$ independent of $\varepsilon \in(0,1]$ and $s, t, u$. Indeed, taking $r=0$, i.e., $\Phi \equiv 1$ we obtain (3.20) with $r_{1}=2, r_{3}=1$. If we take $r=2$, and use the bound $\left|x^{\varepsilon}(t)-x^{\varepsilon}(s)\right| \leqq 2 M$ then we obtain from (3.22) and (3.20)

$$
E\left|x^{\varepsilon}(s)-x^{\varepsilon}(t)\right|^{2}\left|x^{\varepsilon}(u)-x^{\varepsilon}(t)\right|^{2} \leqq C_{1} C(u-s)^{9 / 8} .
$$

In accordance with our suppression of $M$ and previous notation we now write

$$
\begin{aligned}
& \bar{G}(x, \varepsilon)=E G^{M}(x, \varepsilon)=\varphi_{M}(x) E G(x, \varepsilon), \\
& \tilde{G}(x, \varepsilon)=G(x, \varepsilon)-\bar{G}(x, \varepsilon) .
\end{aligned}
$$

Then [see (3.16)]

$$
\begin{aligned}
\left|x^{\varepsilon}(u)-x^{\varepsilon}(t)\right|^{2}= & \frac{2}{\varepsilon} \int_{t}^{u} \sum_{i} G_{i}\left(\frac{\tau}{\varepsilon^{2}}, x^{\varepsilon}(\tau), \varepsilon\right)\left(x_{i}^{\varepsilon}(\tau)-x_{i}^{\varepsilon}(t)\right) d \tau \\
= & \frac{2}{\varepsilon} \int_{t}^{u} \sum_{i} \bar{G}_{i}\left(x^{\varepsilon}(\tau), \varepsilon\right)\left(x_{i}^{\varepsilon}(\tau)-x_{i}^{\varepsilon}(t)\right) d \tau \\
& +\frac{2}{\varepsilon^{2}} \int_{t}^{u} \int_{t}^{\tau} \sum_{j} G_{j}\left(\frac{\sigma}{\varepsilon^{2}}, x^{\varepsilon}(\sigma), \varepsilon\right)\left\{\sum_{i} \tilde{G}_{i, j}\left(\frac{\tau}{\varepsilon^{2}}, x^{\varepsilon}(\sigma), \varepsilon\right)\right. \\
& \left.\cdot\left(x_{i}^{\varepsilon}(\sigma)-x_{i}^{\varepsilon}(t)\right)+\tilde{G}_{j}\left(\frac{\tau}{\varepsilon^{2}}, x^{\varepsilon}(\sigma), \varepsilon\right)\right\} d \sigma d \tau .
\end{aligned}
$$

Here we used the comma-subscript notation for $x$-derivatives. The second equality in (3.24) follows from (3.16) and

$$
\frac{\partial}{\partial x_{j}} \sum_{i} \tilde{G}_{i}\left(\frac{\tau}{\varepsilon^{2}}, x, \varepsilon\right)\left(x_{i}-x_{i}(t)\right)=\sum_{i} \tilde{G}_{i, j}\left(\frac{\tau}{\varepsilon^{2}}, x, \varepsilon\right)\left(x_{i}-x_{i}(t)\right)+\tilde{G}_{j}\left(\frac{\tau}{\varepsilon^{2}}, x, \varepsilon\right) .
$$

It follows from (3.19) and IV that

$$
\begin{aligned}
\mid E & \left\{\frac{2}{\varepsilon} \int_{t}^{u} \sum_{i} \bar{G}_{i}\left(x^{\varepsilon}(\tau), \varepsilon\right)\left(x_{i}^{\varepsilon}(\tau)-x_{i}^{\varepsilon}(t)\right) d \tau \Phi\right\} \mid \\
& \leqq 4 d M(u-t) \frac{1}{\varepsilon} \sup _{|x| \leqq M}|\bar{G}(x, \varepsilon)| E\{|\Phi|\} \\
& \leqq C_{2}(u-t) E^{1 / 8}\left\{\Phi^{8}\right\}
\end{aligned}
$$


for a suitable $C_{2}$. Thus

$$
\begin{gathered}
E\left\{|x(u)-x(t)|^{2} \Phi\right\} \leqq C_{2}(u-t) E^{1 / 8}\left\{\Phi^{8}\right\}+\frac{2}{\varepsilon^{2}} \int_{t}^{u} \int_{t}^{\tau} E\left\{\sum_{j} G_{j}\left(\frac{\sigma}{\varepsilon^{2}}, x^{\varepsilon}(\sigma), \varepsilon\right) \Phi\right. \\
\left.\cdot\left[\sum_{i} \tilde{G}_{i, j}\left(\frac{\tau}{\varepsilon^{2}}, x^{\varepsilon}(\sigma), \varepsilon\right)\left(x_{i}^{\varepsilon}(\sigma)-x_{i}^{\varepsilon}(t)\right)+\tilde{G}_{j}\left(\frac{\tau}{\varepsilon^{2}}, x^{\varepsilon}(\sigma), \varepsilon\right)\right]\right\} d \sigma d \tau .
\end{gathered}
$$

The double integral in (3.26) consists of a number of terms to each of which we can apply the following

Lemma 1. Let $U\left(\frac{t}{\varepsilon^{2}}, x, \varepsilon\right)$ be $\mathscr{G}_{t / \varepsilon^{2}}^{\infty}(\varepsilon)$ measurable for each fixed $|x| \leqq M$, and such that

$$
E\left\{U\left(\frac{t}{\varepsilon^{2}}, x, \varepsilon\right)\right\}=0 .
$$

Let $V\left(\frac{s}{\varepsilon^{2}}, \varepsilon\right), s \leqq t$, be a $\mathscr{G}_{0}^{s / \varepsilon^{2}}(\varepsilon)$ measurable random variable. Then for $0<\gamma \leqq 1$ there exists a constant $C=C(\gamma, d)$ such that for all $0 \leqq \varrho \leqq s \leqq t$

$$
\begin{aligned}
\left|E\left\{U\left(\frac{t}{\varepsilon^{2}}, x^{\varepsilon}(\varrho), \varepsilon\right) V\left(\frac{s}{\varepsilon^{2}}, \varepsilon\right)\right\}\right| \leqq & C\left(E\left\{\sum_{|\beta| \leqq 1} \sup _{|x| \leqq M}\left|D^{\beta} U\left(\frac{t}{\varepsilon^{2}}, x, \varepsilon\right)\right|^{4}\right\}\right)^{1 / 4} \\
& \cdot\left(E\left\{\left|V\left(\frac{s}{\varepsilon^{2}}, \varepsilon\right)\right|^{4}\right\}\right)^{1 / 4}\left\{\beta\left(\frac{t-s}{\varepsilon^{2}}\right)\right\}^{\gamma /(2+\gamma+\gamma d)} .
\end{aligned}
$$

Before proving the lemma we show how we can estimate (3.26) by means of it. For instance we can apply this lemma with

$$
U\left(\frac{\tau}{\varepsilon^{2}}, x, \varepsilon\right)=\tilde{G}_{i, j}\left(\frac{\tau}{\varepsilon^{2}}, x, \varepsilon\right) \in \mathscr{G}_{\tau / \varepsilon^{2}}^{\infty}
$$

and

$$
V\left(\frac{\sigma}{\varepsilon^{2}}, \varepsilon\right)=G_{j}\left(\frac{\sigma}{\varepsilon^{2}}, x^{\varepsilon}(\sigma), \varepsilon\right) \Phi\left(x_{i}^{\varepsilon}(\sigma)-x_{i}^{\varepsilon}(t)\right) .
$$

Since

$$
x^{\varepsilon, M}(\sigma)=x_{0}+\frac{1}{\varepsilon} \int_{0}^{\sigma} G\left(\frac{\varrho}{\varepsilon^{2}}, x^{\varepsilon, M}(\varrho), \varepsilon\right) d \varrho
$$

$x^{\varepsilon, M}(\sigma)$ is $\mathscr{G}_{0}^{\sigma / \varepsilon^{2}}(\varepsilon, M)$ measurable and so is $V\left(\frac{\sigma}{\varepsilon^{2}}, \varepsilon\right)$. Also (3.27) is satisfied because

$$
E\left\{\frac{\partial}{\partial x_{j}} \tilde{G}_{i}\left(\frac{\tau}{\varepsilon^{2}}, x, \varepsilon\right)\right\}=\frac{\partial}{\partial x_{j}} E\left\{\tilde{G}_{i}\left(\frac{\tau}{\varepsilon^{2}}, x, \varepsilon\right)\right\}=0
$$

by virtue of (3.7) and the definition of $\tilde{G}$. Moreover by (3.19)

$$
\left|V\left(\frac{\sigma}{\varepsilon^{2}}, \varepsilon\right)\right| \leqq 2 M|\Phi| \sup _{|x| \leqq M}\left|G\left(\frac{\sigma}{\varepsilon^{2}}, x, \varepsilon\right)\right|
$$


so that Lemma 1 with $\gamma=1$ and Schwarz' inequality and V imply that

$$
\begin{aligned}
& \frac{2}{\varepsilon^{2}}\left|\int_{t}^{u} d \tau \int_{t}^{\tau} d \sigma E\left\{V\left(\frac{\sigma}{\varepsilon^{2}}, \varepsilon\right) U\left(\frac{\tau}{\varepsilon^{2}}, x^{\varepsilon}(\sigma), \varepsilon\right)\right\}\right| \\
& \quad \leqq \frac{C_{3}}{\varepsilon^{2}} E^{1 / 8}\left\{\Phi^{8}\right\} \int_{t}^{u} d \tau \int_{t}^{\tau} d \sigma\left\{\beta\left(\frac{\tau-\sigma}{\varepsilon^{2}}\right)\right\}^{\frac{1}{3+d}} \\
& \leqq C_{3}(u-t) E^{1 / 8}\left\{\Phi^{8}\right\} \int_{0}^{\infty}\{\beta(s)\}^{\frac{1}{3+d}} d s \\
& \leqq C_{4}(u-t) E^{1 / 8}\left\{\Phi^{8}\right\} .
\end{aligned}
$$

The other terms in (3.26) are handled similarly.

Thus (3.22) and the tightness of $\left\{Q^{\varepsilon}\right\}_{0<\varepsilon \leqq 1}$ have been reduced to Lemma 1.

Step (iv). Proof of Lemma 1. First we approximate $x^{\varepsilon}(\varrho, \omega)$ by step functions as follows: For $\omega$ in the set

$$
A(k)=\left\{\omega: \frac{k_{j}}{N} M \leqq x_{j}^{\varepsilon}(\varrho, \omega)<\frac{\left(k_{j}+1\right)}{N} M, \quad 1 \leqq j \leqq d\right\}
$$

take

$$
x_{i}^{\varepsilon, N}(\varrho, \omega)= \begin{cases}\frac{k_{i}}{N} M & \text { if } \quad k_{i} \geqq 0, \\ \frac{\left(k_{i}+1\right)}{N} M & \text { if } \quad k_{i}<0 .\end{cases}
$$

For brevity we denote the right hand side of (3.29) by $\bar{k} M / N$. Clearly

$$
\left|x_{i}^{\varepsilon, N}(\varrho, \omega)-x_{i}^{\varepsilon}(\varrho, \omega)\right| \leqq \frac{M}{N}
$$

and

$$
\left|x^{\varepsilon, N}(\varrho, \omega)\right| \leqq \mid x^{\varepsilon}(\varrho, \omega) \leqq M \quad \text { for all } \omega .
$$

Moreover $A(k) \in \mathscr{G}_{0}^{s / \varepsilon^{2}}(\varepsilon)$ since $x^{\varepsilon}(\varrho)$ is $\mathscr{G}_{0}^{\varrho / \varepsilon^{2}}(\varepsilon)$ measurable. Define also

$$
U_{K}=\left\{\begin{array}{lll}
U & \text { if } & |U| \leqq K \\
0 & \text { if } & |U|>K
\end{array}\right.
$$

and similarly for $V_{L}$ for $V$ truncated at $L$. Then

$$
\begin{aligned}
\mid E & \left\{U\left(\frac{t}{\varepsilon^{2}}, x^{\varepsilon}(\varrho), \varepsilon\right) V\left(\frac{s}{\varepsilon^{2}}, \varepsilon\right)\right\} \mid \\
& \leqq\left|E\left\{\left(U\left(\frac{t}{\varepsilon^{2}}, x^{\varepsilon}(\varrho), \varepsilon\right)-U\left(\frac{t}{\varepsilon^{2}}, x^{\varepsilon, N}(\varrho), \varepsilon\right)\right) V\left(\frac{s}{\varepsilon^{2}}, \varepsilon\right)\right\}\right| \\
& +\left|E\left\{\left(U\left(\frac{t}{\varepsilon^{2}}, x^{\varepsilon, N}(\varrho), \varepsilon\right)-U_{K}\left(\frac{t}{\varepsilon^{2}}, x^{\varepsilon, N}(\varrho), \varepsilon\right)\right) V\left(\frac{s}{\varepsilon^{2}}, \varepsilon\right)\right\}\right|
\end{aligned}
$$




$$
\begin{aligned}
& +\left|E\left\{U_{K}\left(\frac{t}{\varepsilon^{2}}, x^{\varepsilon, N}(\varrho), \varepsilon\right)\left(V\left(\frac{s}{\varepsilon^{2}}, \varepsilon\right)-V_{L}\left(\frac{s}{\varepsilon^{2}}, \varepsilon\right)\right)\right\}\right| \\
& +\sum_{k}\left|E\left\{U_{K}\left(\frac{t}{\varepsilon^{2}}, \frac{\bar{k} M}{N}, \varepsilon\right) \chi_{A(k)} V_{L}\left(\frac{s}{\varepsilon^{2}}, \varepsilon\right)\right\}\right| .
\end{aligned}
$$

Here $k$ stands for an integral $k$-vector $\left(k_{1}, \ldots, k_{d}\right)$ and the sum over $k$ runs over the $(2 N+1)^{d}$ such vectors with $\left|k_{j}\right| \leqq N, \chi_{A}$ stands for the indicator function of $A$. The first-third term are estimated in the obvious way by means of (3.30) without intervention of the mixing hypothesis. In the last term in the right hand side of (3.31) we apply Theorem 17.2.1 of [7] to each summand. Since $\left|U_{K}\right| \leqq K,\left|V_{L}\right| \leqq L$, we find that the $k$-term is bounded by

$$
\begin{aligned}
& 4 K L \beta\left(\frac{t-s}{\varepsilon^{2}}\right)+\left|E\left\{U_{K}\left(\frac{t}{\varepsilon^{2}}, \frac{\bar{k} M}{N}, \varepsilon\right)\right\} E\left\{\chi_{A(k)} V_{L}\left(\frac{s}{\varepsilon^{2}}, \varepsilon\right)\right\}\right| \\
& \leqq 4 K L \beta\left(\frac{t-s}{\varepsilon^{2}}\right)+\frac{1}{K^{\gamma}} E\left\{\sup _{|x| \leqq M}\left|U\left(\frac{t}{\varepsilon^{2}}, x, \varepsilon\right)\right|^{1+\gamma}\right\} E\left\{\chi_{A(k)}\left|V\left(\frac{s}{\varepsilon^{2}}, \varepsilon\right)\right|\right\}
\end{aligned}
$$

[use (3.27) to estimate $E\left\{U_{K}\right\}$ ]. After summing over $k$ we obtain that the left hand side of (3.28) is at most

$$
\begin{aligned}
C_{5} & {\left[\frac{1}{N} E\left\{\sum_{|\beta|=1} \sup _{|x| \leqq M}\left|D^{\beta} U\left(\frac{t}{\varepsilon^{2}}, x, \varepsilon\right)\right|\left|V\left(\frac{s}{\varepsilon^{2}}, \varepsilon\right)\right|\right\}\right.} \\
+ & \frac{1}{K^{\gamma}} E\left\{\sup _{|x| \leqq M}\left|U\left(\frac{t}{\varepsilon^{2}}, x, \varepsilon\right)\right|^{1+\gamma}\left|V\left(\frac{s}{\varepsilon^{2}}, \varepsilon\right)\right|\right\} \\
+ & \frac{1}{L^{\gamma}} E\left\{\left.\sup _{|x| \leqq M}\left|U\left(\frac{t}{\varepsilon^{2}}, x, \varepsilon\right)\right| V\left(\frac{s}{\varepsilon^{2}}, \varepsilon\right)\right|^{1+\gamma}\right\} \\
+ & \frac{1}{K^{\gamma}} E\left\{\sup _{|x| \leqq M}\left|U\left(\frac{t}{\varepsilon^{2}}, x, \varepsilon\right)\right|^{1+\gamma}\right\} E\left\{\left|V\left(\frac{s}{\varepsilon^{2}}, \varepsilon\right)\right|\right\} \\
+ & \left.K L(N+1)^{d} \beta\left(\frac{t-s}{\varepsilon^{2}}\right)\right] .
\end{aligned}
$$

(3.28) now follows from Schwarz' and Jensens' inequalities if we take

$$
\begin{aligned}
& K=\left(E\left\{\sum_{|\beta| \leqq 1} \sup _{|x| \leqq M}\left|U\left(\frac{t}{\varepsilon}, x, \varepsilon\right)\right|^{4}\right\}\right)^{1 / 4}\left\{(N+1)^{d} \beta\left(\frac{t-s}{\varepsilon}\right)\right\}^{-\frac{1}{2+\gamma}}, \\
& L=\left(E\left\{\left|V\left(\frac{s}{\varepsilon}, \varepsilon\right)\right|^{4}\right\}\right)^{1 / 4}\left\{(N+1)^{d} \beta\left(\frac{t-s}{\varepsilon^{2}}\right)\right\}^{-\frac{1}{2+\gamma}}, \\
& N=\left[\left\{\beta\left(\frac{t-s}{\varepsilon^{2}}\right)\right\}^{-\gamma /(2+\gamma+\gamma d)}\right] \geqq 1 \quad(\text { since } \beta \leqq 1) .
\end{aligned}
$$


For the next step we also need the following variant of Lemma 1.

Lemma 2. Let $X\left(\frac{s}{\varepsilon^{2}}, \varepsilon\right)$ be $\mathscr{G}_{0}^{s / \varepsilon^{2}}(\varepsilon)$ measurable, and for $|x| \leqq M$, let $U\left(\frac{t}{\varepsilon^{2}}, x, \varepsilon\right)\left(V\left(\frac{u}{\varepsilon^{2}}, x, \varepsilon\right)\right)$ be $\mathscr{G}_{t / \varepsilon^{2}}^{t / \varepsilon^{2}}\left(\right.$ respectively $\left.\mathscr{G}_{u / \varepsilon^{2}}^{u / \varepsilon^{2}}(\varepsilon)\right)$ measurable. Assume that

$$
E\left\{V\left(\frac{u}{\varepsilon^{2}}, x, \varepsilon\right)\right\}=0
$$

and set

$$
W\left(\frac{t}{\varepsilon^{2}}, \frac{u}{\varepsilon^{2}}, x, \varepsilon\right)=E\left\{U\left(\frac{t}{\varepsilon^{2}}, x, \varepsilon\right) V\left(\frac{u}{\varepsilon^{2}}, x, \varepsilon\right)\right\} .
$$

Then for each $0<\gamma \leqq 1$ there exists a constant $C=C(\gamma, d)$ such that for all $0 \leqq s \leqq t \leqq u$

$$
\begin{aligned}
&\left|E\left\{X\left(\frac{s}{\varepsilon^{2}}, \varepsilon\right)\left[U\left(\frac{t}{\varepsilon^{2}}, x^{\varepsilon}(s), \varepsilon\right) V\left(\frac{u}{\varepsilon^{2}}, x^{\varepsilon}(s), \varepsilon\right)-W\left(\frac{t}{\varepsilon^{2}}, \frac{u}{\varepsilon^{2}}, x^{\varepsilon}(s), \varepsilon\right)\right]\right\}\right| \\
& \leqq C\left(E\left\{\left|X\left(\frac{s}{\varepsilon^{2}}, \varepsilon\right)\right|^{8}\right\}\right)^{1 / 8}\left(E\left\{\sum_{|\beta| \leqq 1} D^{\beta} \sup _{|x| \leqq M}\left|U\left(\frac{t}{\varepsilon^{2}}, x, \varepsilon\right)\right|^{8}\right\}\right)^{1 / 8} \\
& \cdot\left(E\left\{\sum_{|\beta| \leqq 1} D^{\beta} \sup _{|x| \leqq M}\left|V\left(\frac{u}{\varepsilon^{2}}, x, \varepsilon\right)\right|^{8}\right\}\right)^{1 / 8} \\
& \cdot\left\{\beta\left(\frac{t-s}{\varepsilon^{2}}\right) \beta\left(\frac{u-t}{\varepsilon^{2}}\right)\right\}^{\gamma /(4+2 \gamma+2 \gamma d)} .
\end{aligned}
$$

Proof. First apply Lemma 1 with $U V-W$ substituted for $U$ and $X$ for $V$. This shows that the left hand side of (3.33) is bounded by

$$
\begin{aligned}
& C\left(E\left\{\sum_{|\beta| \leqq 1} \sup _{|x| \leqq M}\left|D^{\beta} U\left(\frac{t}{\varepsilon^{2}}, x, \varepsilon\right) V\left(\frac{u}{\varepsilon^{2}}, x, \varepsilon\right)-D^{\beta} W\left(\frac{t}{\varepsilon^{2}}, \frac{u}{\varepsilon^{2}}, x, \varepsilon\right)\right|^{4}\right\}\right)^{1 / 4} \\
& \cdot\left(E\left\{\left|X\left(\frac{s}{\varepsilon^{2}}, \varepsilon\right)\right|^{4}\right\}\right)^{1 / 4}\left\{\beta\left(\frac{t-s}{\varepsilon^{2}}\right)\right\}^{\gamma /(2+\gamma+\gamma d)} \\
& \leqq C^{*}\left(E\left\{\sum_{|\beta| \leqq 1} \sup _{|x| \leqq M}\left|D^{\beta} U\left(\frac{t}{\varepsilon^{2}}, x, \varepsilon\right)\right|^{8}\right\}\right)^{1 / 8} \\
&\left.\cdot\left(E\left\{\sum_{|\beta| \leqq 1} \sup _{|x| \leqq M}\left|D^{\beta} V\left(\frac{u}{\varepsilon^{2}}, x, \varepsilon\right)\right|^{8}\right\}\right)^{1 / 8}\right) \\
& \cdot\left(E\left\{\left|X\left(\frac{s}{\varepsilon^{2}}, \varepsilon\right)\right|^{4}\right\}\right)^{1 / 4}\left\{\beta\left(\frac{t-s}{\varepsilon^{2}}\right)\right\}^{\gamma /(2+\gamma+\gamma d)} .
\end{aligned}
$$


Next we apply Lemma 1 again, this time with $V$ substituted for $U$ and $X U$ for $V$. We find that

$$
\begin{aligned}
&\left|E\left\{X\left(\frac{s}{\varepsilon^{2}}, \varepsilon\right) U\left(\frac{t}{\varepsilon^{2}}, x^{\varepsilon}(s), \varepsilon\right) V\left(\frac{u}{\varepsilon^{2}}, x^{\varepsilon}(s), \varepsilon\right)\right\}\right| \\
& \leqq C\left(E\left\{\sum_{|\beta| \leqq 1} \sup _{|x| \leqq M}\left|D^{\beta} V\left(\frac{u}{\varepsilon^{2}}, x, \varepsilon\right)\right|^{4}\right\}\right)^{1 / 4} \\
& \cdot\left(E\left\{\left|X\left(\frac{s}{\varepsilon^{2}}, \varepsilon\right)\right|^{8}\right\} E\left\{\sup _{|x| \leqq M}\left|U\left(\frac{t}{\varepsilon^{2}}, x, \varepsilon\right)\right|^{8}\right\}\right)^{1 / 8} \\
& \cdot\left\{\beta\left(\frac{u-t}{\varepsilon^{2}}\right)\right\}^{\gamma /(2+\gamma+\gamma d)} .
\end{aligned}
$$

Lastly, by Theorem 17.2.2 of [7], p. 307

$$
\begin{aligned}
& \left|W\left(\frac{t}{\varepsilon^{2}}, \frac{u}{\varepsilon^{2}}, x^{\varepsilon}(s), \varepsilon\right)\right| \leqq \sup _{|x| \leqq M}\left|E\left\{U\left(\frac{t}{\varepsilon^{2}}, x, \varepsilon\right) V\left(\frac{u}{\varepsilon^{2}}, x, \varepsilon\right)\right\}\right| \\
& \leqq C \sup _{|x| \leqq M}\left(E\left\{\left|U\left(\frac{t}{\varepsilon^{2}}, x, \varepsilon\right)\right|^{4}\right\}^{1 / 4} E\left\{\left|V\left(\frac{t}{\varepsilon^{2}}, x, \varepsilon\right)\right|^{4}\right\}\right)^{1 / 4}\left\{\beta\left(\frac{u-t}{\varepsilon^{2}}\right)\right\}^{1 / 3} .
\end{aligned}
$$

Since $\beta \leqq 1$ and $\frac{1}{3} \geqq \gamma /(2+\gamma+\gamma d)$ for $\gamma \leqq 1$, we see that the left hand side of (3.33) is also bounded by twice the right hand side of (3.35). Thus the square of the left hand side of (3.33) is bounded by twice the product of the last member of (3.34) and (3.35). This fact plus another application of Jensen's inequality proves (3.33).

Step $(v)$. This is the first step in the identification of the possible limit points of $R^{\varepsilon}$ as $\varepsilon \downarrow 0$. We reintroduce the superscript $M$. As in Step (iii) $Q^{\varepsilon, M}$ is the measure on $D=D\left([0, \infty) ; \mathbb{R}^{d}\right)$ induced by $x^{\varepsilon, M} \cdot X(t)$ will be the $t$-coordinate function on $D$ and the corresponding $\sigma$-fields of subsets of $D$ are given by

$$
\mathscr{M}_{u}^{v}=\sigma \text {-field generated by }\left\{X_{t}: u \leqq t \leqq v\right\} \text {. }
$$

Assume now that $\left\{\varepsilon_{n}\right\}$ is a sequence of positive numbers tending to zero such that

$$
Q^{\varepsilon_{n}, M} \Rightarrow Q^{M} \quad \text { as } n \rightarrow \infty .
$$

(As usual $\Rightarrow$ here means weak convergence on $D\left([0, \infty) ; \mathbb{R}^{d}\right.$ ) with its $J_{1}$ Skorokhod topology; see [5], Chap. 3 and [6] for details.) Expectations with respect to $Q^{M}$ will be denoted by $E^{M}$. For any $C^{\infty}$ function $f: \mathbb{R}^{d} \rightarrow \mathbb{R}$ with compact support define

$$
L^{M} f(x)=\frac{1}{2} \sum_{i, j=1}^{d} a_{i j}^{M}(x) \frac{\partial^{2} f(x)}{\partial x_{i} \partial x_{j}}+\sum_{j=1}^{d} b_{j}^{M}(x) \frac{\partial f(x)}{\partial x_{j}},
$$

where

$$
\begin{aligned}
& a_{i j}^{M}(x)=a_{i j}(x) \varphi_{M}^{2}(x), \quad c_{i j}^{M}(x)=c_{i j}(x) \varphi_{M}^{2}(x)+\varphi_{M}(x)\left(\frac{\partial}{\partial x_{i}} \varphi_{M}(x)\right) A_{i j}(x), \\
& b_{j}^{M}(x)=\sum_{i} c_{i j}^{M}(x)+w_{j} \varphi_{M}(x)
\end{aligned}
$$


We show in this step that

$$
f(X(t))-\int_{0}^{t} L^{M} f(X(\sigma)) d \sigma
$$

is a $\left(Q^{M}, \mathscr{M}_{0}^{t}\right)$ martingale.

To prove that (3.40) is a martingale it suffices to prove for any integer $m>0$ and bounded continuous function $\Phi:\left(\mathbb{R}^{d}\right)^{m} \rightarrow \mathbb{R}$ and $0 \leqq s_{1}<s_{2}<\ldots<s_{m} \leqq s \leqq t$ that

$$
\begin{aligned}
E^{M} & \left\{(f(X(t))-f(X(s))) \Phi\left(X\left(s_{1}\right), \ldots, X\left(s_{m}\right)\right)\right\} \\
= & E^{M}\left\{\int_{s}^{t} L^{M} f(X(\sigma)) \Phi\left(X\left(s_{1}\right), \ldots, X\left(s_{m}\right)\right) d \sigma\right\} .
\end{aligned}
$$

Indeed, a standard monotone class argument shows that (3.41) implies

$$
E^{M}\{f(X(t)) \Phi\}=E^{M}\{f(X(s)) \Phi\}+E^{M}\left\{\int_{s}^{t} L^{M} f(X(\sigma)) \Phi d \sigma\right\}
$$

for any bounded $\mathscr{M}_{0}^{s}$ measurable function $\Phi$. This is clearly equivalent to (3.40) being a martingale.

In addition,

$$
\left(f\left(x^{\varepsilon, M}(t)\right)-f\left(x^{\varepsilon, M}(s)\right)\right) \Phi\left(x^{\varepsilon, M}\left(s_{1}\right), \ldots, x^{\varepsilon, M}\left(s_{m}\right)\right)
$$

is bounded and because $X \rightarrow f(X(s))$ and $X \rightarrow \Phi\left(X\left(s_{1}\right), \ldots, X\left(s_{m}\right)\right)$ are continuous a.e. $\left[Q^{M}\right]$ on $D$ [by (3.20) and (3.37)], it converges in distribution to

$$
(f(X(t))-f(X(s))) \Phi\left(X\left(s_{1}\right), \ldots, X\left(s_{m}\right)\right)
$$

as $\varepsilon \rightarrow 0$ through the sequence $\left\{\varepsilon_{n}\right\}$. Thus the left hand side of (3.41) equals

$$
\lim _{n \rightarrow \infty} E\left\{\left(f\left(x^{\varepsilon_{n}, M}(t)\right)-f\left(x^{\varepsilon_{n}, M}(s)\right)\right) \Phi\left(x^{\varepsilon_{n}, M}\left(s_{1}\right), \ldots, x^{\varepsilon_{n}, M}\left(s_{m}\right)\right)\right\} .
$$

Now with $\bar{G}^{M}(x, \varepsilon)=\bar{G}(x, \varepsilon) \varphi_{M}(x)$ and $\tilde{G}^{M}=G^{M}-\bar{G}^{M}$, as in (3.23), we have analogously to (3.24)

$$
\begin{aligned}
f\left(x^{\varepsilon, M}(t)\right)-f\left(x^{\varepsilon, M}(s)\right) \\
=\frac{1}{\varepsilon} \int_{s}^{t} \sum_{i} \frac{\partial f}{\partial x_{i}}\left(x^{\varepsilon, M}(\tau)\right) G_{i}^{M}\left(\frac{\tau}{\varepsilon^{2}}, x^{\varepsilon, M}(\tau), \varepsilon\right) d \tau \\
=\frac{1}{\varepsilon} \int_{s}^{t} \sum_{i} \frac{\partial f}{\partial x_{i}}\left(x^{\varepsilon, M}(\tau)\right) \bar{G}_{i}^{M}\left(x^{\varepsilon, M}(\tau), \varepsilon\right) d \tau \\
\quad+\frac{1}{\varepsilon} \int_{s}^{t} \sum_{i} \frac{\partial f}{\partial x_{i}}\left(x^{\varepsilon, M}(s)\right) \tilde{G}_{i}^{M}\left(\frac{\tau}{\varepsilon^{2}}, x^{\varepsilon, M}(s), \varepsilon\right) d \tau \\
\quad+\frac{1}{\varepsilon^{2}} \int_{s}^{t} d \tau \int_{s}^{\tau} d \sigma \sum_{i j}\left[\frac{\partial^{2} f}{\partial x_{j} \partial x_{i}}\left(x^{\varepsilon, M}(\sigma)\right) \tilde{G}_{i}^{M}\left(\frac{\tau}{\varepsilon^{2}}, x^{\varepsilon, M}(\sigma), \varepsilon\right)\right. \\
\left.\quad+\frac{\partial f}{\partial x_{i}}\left(x^{\varepsilon, M}(\sigma)\right) \tilde{G}_{i, j}^{M}\left(\frac{\tau}{\varepsilon^{2}}, x^{\varepsilon, M}(\sigma), \varepsilon\right)\right] G_{j}^{M}\left(\frac{\sigma}{\varepsilon^{2}}, x^{\varepsilon, M}(\sigma), \varepsilon\right) .
\end{aligned}
$$


We now multiply (3.44) with $\Phi$ and take expectations and let $\varepsilon \downarrow 0$ through the sequence $\varepsilon_{n}$. The first term in the right hand side yields

$$
\begin{gathered}
\lim \int_{s}^{t} \sum_{i} E\left\{\frac{\partial f}{\partial x_{i}}\left(x^{\varepsilon, M}(\tau)\right) \frac{1}{\varepsilon} \bar{G}_{i}^{M}\left(x^{\varepsilon, M}(\tau), \varepsilon\right) \Phi\right\} d \tau \\
=E^{M}\left\{\int_{s}^{t} \sum_{i} \frac{\partial f}{\partial x_{i}}(X(\tau)) w_{i} \varphi_{M}(X(\tau)) \Phi\right\} d \tau
\end{gathered}
$$

by virtue of IV and (3.37). The second term has limit zero, on account of Lemma 1. Indeed, an application of Lemma 1 with $U\left(\frac{\tau}{\varepsilon^{2}}, x, \varepsilon\right)=\tilde{G}_{i}^{M}\left(\frac{\tau}{\varepsilon^{2}}, x, \varepsilon\right)$ [note that this has expectation zero by definition of $\bar{G}$, cf. (3.5)] and $V\left(\frac{s}{\varepsilon^{2}}, \varepsilon\right)=\Phi \frac{\partial f}{\partial x_{i}}\left(x^{\varepsilon, M}(s)\right)$, $\gamma=1$ shows that

$$
\begin{gathered}
\frac{1}{\varepsilon} \int_{s}^{t} \sum_{i} E\left\{\frac{\partial f}{\partial x_{i}}\left(x^{\varepsilon, M}(s)\right) \tilde{G}_{i}^{M}\left(\frac{\tau}{\varepsilon^{2}}, x^{\varepsilon, M}(s), \varepsilon\right) \Phi\right\} d \tau \\
=O\left(\frac{1}{\varepsilon} \int_{s}^{t}\left\{\beta\left(\frac{\tau-s}{\varepsilon^{2}}\right)\right\}^{1 /(3+d)} d \tau\right)=O(\varepsilon) .
\end{gathered}
$$

Finally the double integral in the right hand side of (3.44) is handled by means of Lemma 2. We restrict ourselves to the term

$$
\begin{gathered}
\frac{1}{\varepsilon^{2}} \int_{s}^{t} d \tau \int_{s}^{t} d \sigma E\left\{\frac{\partial^{2} f}{\partial x_{j} \partial x_{i}}\left(x^{\varepsilon, M}(\sigma)\right) \tilde{G}_{i}^{M}\left(\frac{\tau}{\varepsilon^{2}}, x^{\varepsilon, M}(\sigma), \varepsilon\right)\right. \\
\left.\cdot G_{j}^{M}\left(\frac{\sigma}{\varepsilon^{2}}, x^{\varepsilon, M}(\sigma), \varepsilon\right) \Phi\right\} .
\end{gathered}
$$

We write

$$
H\left(\frac{\tau}{\varepsilon^{2}}, \frac{\sigma}{\varepsilon^{2}}, x, \varepsilon\right)=\tilde{G}_{i}^{M}\left(\frac{\tau}{\varepsilon^{2}}, x, \varepsilon\right) G_{j}^{M}\left(\frac{\sigma}{\varepsilon^{2}}, x, \varepsilon\right)
$$

and in accordance with previous notation put

$$
\begin{aligned}
\bar{H}\left(\frac{\tau}{\varepsilon^{2}}, \frac{\sigma}{\varepsilon^{2}}, x, \varepsilon\right) & =E H\left(\frac{\tau}{\varepsilon^{2}}, \frac{\sigma}{\varepsilon^{2}}, x, \varepsilon\right), \\
\tilde{H} & =H-\bar{H} .
\end{aligned}
$$

Then (3.47) equals

$$
\begin{aligned}
& \frac{1}{\varepsilon^{2}} \int_{s}^{t} d \tau \int_{s}^{\tau} d \sigma E\left\{\frac{\partial^{2} f}{\partial x_{j} \partial x_{i}}\left(x^{\varepsilon, M}(\sigma)\right) \bar{H}\left(\frac{\tau}{\varepsilon^{2}}, \frac{\sigma}{\varepsilon^{2}}, x^{\varepsilon, M}(\sigma), \varepsilon\right) \Phi\right\} \\
& \quad+\frac{1}{\varepsilon^{2}} \int_{s}^{t} d \tau \int_{s}^{\tau} d \sigma E\left\{\frac{\partial^{2} f}{\partial x_{j} \partial x_{i}}\left(x^{\varepsilon, M}(\sigma)\right) \tilde{H}\left(\frac{\tau}{\varepsilon^{2}}, \frac{\sigma}{\varepsilon^{2}}, x^{\varepsilon, M}(\sigma), \varepsilon\right) \Phi\right\} .
\end{aligned}
$$


We first analyze the $\bar{H}$ term in (3.48); as we shall see this contributes the part of

$$
E^{M}\left\{\int_{s}^{t} d \sigma L^{M} f(X(\sigma)) \Phi\right\}
$$

with the second derivatives. Indeed, after a change in the order of integration the $\bar{H}$ term in (3.48) becomes

$$
\begin{aligned}
& \int_{s}^{t} d \sigma E\left\{\Phi \frac{\partial^{2} f}{\partial x_{j} \partial x_{i}}\left(x^{\varepsilon, M}(\sigma)\right)\right. \\
& \left.\cdot \int_{\sigma / \varepsilon^{2}}^{t / \varepsilon^{2}} E\left\{G_{j}^{M}\left(\frac{\sigma}{\varepsilon^{2}}, x, \varepsilon\right) \tilde{G}_{i}^{M}(\lambda, x, \varepsilon)\right\}_{x=x^{\varepsilon, M}(\sigma)} d \lambda\right\} .
\end{aligned}
$$

By the standard mixing Theorem 17.2.2 of [7]

$$
\left|E\left\{G_{j}^{M}\left(\frac{\sigma}{\varepsilon^{2}}, x, \varepsilon\right) \tilde{G}_{i}^{M}(\lambda, x, \varepsilon)\right\}\right| \leqq C\left\{\beta\left(\lambda-\frac{\sigma}{\varepsilon^{2}}\right)\right\}^{1 / 3},
$$

uniformly in $|x| \leqq M, \varepsilon \in(0,1]$. Thus by II and (3.8)

$$
\begin{aligned}
\int_{\sigma / \varepsilon^{2}}^{t / \varepsilon^{2}} E & \left\{G_{j}^{M}\left(\frac{\sigma}{\varepsilon^{2}}, x, \varepsilon\right) \tilde{G}_{i}^{M}(\lambda, x, \varepsilon)\right\} d \lambda \\
= & \varphi_{M}^{2}(x) \int_{0}^{\infty} E\left\{G_{j}(0, x, \varepsilon) \tilde{G}_{i}(\lambda, x, \varepsilon)\right\} d \lambda \\
& +O\left(\int_{t / \varepsilon^{2}}^{\infty}\left\{\beta\left(\lambda-\frac{\sigma}{\varepsilon^{2}}\right)\right\}^{1 / 3} d \lambda\right) \\
= & \varphi_{M}^{2}(x) A_{j i}(x)+o_{\varepsilon}(1)
\end{aligned}
$$

as $\varepsilon \downarrow 0$, uniformly in $|x| \leqq M$, for each fixed $\sigma<t$. It follows that the limit of (3.49) as $\varepsilon \downarrow 0$ through the sequence $\varepsilon_{n}$ equals

$$
\begin{gathered}
\lim E\left\{\int_{s}^{t} \Phi \frac{\partial^{2} f}{\partial x_{j} \partial x_{i}}\left(x^{\varepsilon, M}(\sigma)\right) A_{j i}^{M}\left(x^{\varepsilon, M}(\sigma)\right) d \sigma\right\} \\
=E^{M}\left\{\int_{s}^{t} \Phi A_{j i}^{M}(X(\sigma)) \frac{\partial^{2} f}{\partial x_{j} \partial x_{i}}(X(\sigma)) d \sigma\right\}
\end{gathered}
$$

as claimed [again we used (3.37)] and $A_{j i}^{M}=\varphi_{M}^{2} A_{j i}$.

Next we shall show that the $\tilde{H}$ term in (3.48) tends to zero. For this we need to apply (3.16) once more to obtain

$$
\begin{aligned}
& \int_{s}^{t} d \tau \int_{s}^{\tau} d \sigma E\left\{\frac{\partial^{2} f}{\partial x_{j} \partial x_{i}}\left(x^{\varepsilon, M}(\sigma)\right) \tilde{H}\left(\frac{\tau}{\varepsilon^{2}}, \frac{\sigma}{\varepsilon^{2}}, x^{\varepsilon, M}(\sigma), \varepsilon\right) \Phi\right\} \\
& =\int_{s}^{t} d \tau \int_{s}^{\tau} d \sigma E\left\{\frac{\partial^{2} f}{\partial x_{j} \partial x_{i}}\left(x^{\varepsilon, M}(s)\right) \tilde{H}\left(\frac{\tau}{\varepsilon^{2}}, \frac{\sigma}{\varepsilon^{2}}, x^{\varepsilon, M}(s), \varepsilon\right) \Phi\right\} \\
& \quad+\frac{1}{\varepsilon} \sum_{k} \int_{s}^{t} d \tau \int_{s}^{\tau} d \sigma \int_{s}^{\sigma} d \lambda E\left\{\left[\frac{\partial^{3} f}{\partial x_{k} \partial x_{j} \partial x_{i}}\left(x^{\varepsilon, M}(\lambda)\right) \tilde{H}\left(\frac{\tau}{\varepsilon^{2}}, \frac{\sigma}{\varepsilon^{2}}, x^{\varepsilon, M}(\lambda), \varepsilon\right)\right.\right.
\end{aligned}
$$




$$
\begin{aligned}
& \left.+\frac{\partial^{2} f}{\partial x_{j} \partial x_{i}}\left(x^{\varepsilon, M}(\lambda)\right) \frac{\partial}{\partial x_{k}} \tilde{H}\left(\frac{\tau}{\varepsilon^{2}}, \frac{\sigma}{\varepsilon^{2}}, x^{\varepsilon, M}(\lambda), \varepsilon\right)\right] \\
& \left.\cdot G_{k}\left(\frac{\lambda}{\varepsilon^{2}}, x^{\varepsilon, M}(\lambda), \varepsilon\right) \Phi\right\} .
\end{aligned}
$$

First estimate the double integral in the right hand side of (3.51) by means of Lemma 2. Take

$$
\begin{aligned}
X\left(\frac{s}{\varepsilon^{2}}, \varepsilon\right) & =\frac{\partial^{2} f}{\partial x_{j} \partial x_{i}}\left(x^{\varepsilon, M}(s)\right) \Phi, \\
U\left(\frac{\sigma}{\varepsilon^{2}}, x, \varepsilon\right) & =G_{j}^{M}\left(\frac{\sigma}{\varepsilon^{2}}, x, \varepsilon\right), \\
V\left(\frac{\tau}{\varepsilon^{2}}, x, \varepsilon\right) & =\tilde{G}_{i}^{M}\left(\frac{\tau}{\varepsilon^{2}}, x, \varepsilon\right) .
\end{aligned}
$$

Then by Lemma 2 with $\gamma=1$ we find that the double integral is

$$
O\left(\left\{\beta\left(\frac{\tau-\sigma}{\varepsilon^{2}}\right) \beta\left(\frac{\sigma-s}{\varepsilon^{2}}\right)\right\}^{\frac{1}{6+2 d}}\right)
$$

and by (3.4)

$$
\frac{1}{\varepsilon^{2}} \int_{s}^{t} d \tau \int_{s}^{\tau} d \sigma\left\{\beta\left(\frac{\tau-\sigma}{\varepsilon^{2}}\right) \beta\left(\frac{\sigma-s}{\varepsilon^{2}}\right)\right\}^{\frac{1}{6+2 d}}=O\left(\varepsilon^{2}\right) .
$$

The triple integral in (3.51) has two terms. We estimate the first. This time take

$$
X\left(\frac{\lambda}{\varepsilon^{2}}, \varepsilon\right)=\frac{\partial^{3} f}{\partial x_{k} \partial x_{j} \partial x_{i}}\left(x^{\varepsilon, M}(\lambda)\right) G_{k}\left(\frac{\lambda}{\varepsilon^{2}}, x^{\varepsilon, M}(\lambda), \varepsilon\right) \Phi,
$$

and $U, V$ as in (3.52). By Lemma 2 this term becomes

$$
O\left(\frac{1}{\varepsilon} \int_{s}^{t} d \tau \int_{s}^{\tau} d \sigma \int_{s}^{\sigma} d \lambda\left\{\beta\left(\frac{\tau-\sigma}{\varepsilon^{2}}\right) \beta\left(\frac{\sigma-\lambda}{\varepsilon^{2}}\right)\right\}^{\frac{1}{6+2 d}}\right)=O\left(\varepsilon^{3}\right) .
$$

Thus again $\varepsilon^{-2}$ times this term tends to zero and similarly for the second term in the triple integral.

The above observations show that the limit of (3.47) is given by (3.50). In the same way we obtain

$$
\begin{gathered}
\frac{1}{\varepsilon^{2}} \int_{s}^{t} d \tau \int_{s}^{\tau} d \sigma E\left\{\sum_{i, j} \frac{\partial f}{\partial x_{i}}\left(x^{\varepsilon, M}(\sigma)\right) \tilde{G}_{i, j}^{M}\left(\frac{\tau}{\varepsilon^{2}}, x^{\varepsilon, M}(\sigma), \varepsilon\right)\right. \\
\left.\cdot G_{j}^{M}\left(\frac{\sigma}{\varepsilon^{2}}, x^{\varepsilon, M}(\sigma), \varepsilon\right) \Phi\right\} \\
\rightarrow E^{M}\left\{\int_{s}^{t} \Phi \sum_{i, j} \frac{\partial f}{\partial x_{i}}(X(\sigma)) c_{j i}^{M}(X(\sigma))\right\} .
\end{gathered}
$$


Adding all the contributions we finally obtain that (3.43) equals

$$
E^{M}\left\{\int_{s}^{t} L^{M} f(X(\sigma)) \Phi d \sigma\right\}
$$

as required.

Step (vi). In this step we remove the cutoff in $M$ and show that as $M \rightarrow \infty$ the measures $Q^{M}$ of the last step converge to $R$. Because of the tightness of the family $\left\{Q^{\varepsilon, M}: 0<\varepsilon \leqq 1\right\}$ [see Step (iii)] each sequence of $\varepsilon_{n} \downarrow 0$ contains a subsequence for which (3.37) holds for some $Q^{M}$. If we could show uniqueness of $Q^{M}$ then we would have $Q^{\varepsilon, M} \Rightarrow Q^{M}$ as $\varepsilon \rightarrow 0$ in any way. Because the coefficients of $L^{M}$ vanish for large $x$ it is somewhat unpleasant to prove uniqueness for $Q^{M}$. Another nuisance difficulty is that $Q^{\varepsilon, M}$ and $Q^{M}$ are measures on $D=D\left([0, \infty) ; \mathbb{R}^{d}\right)$ rather than on $C=C\left([0, \infty) ; \mathbb{R}^{d}\right)$. Since $x^{\varepsilon, M}(t)$ is continuous, $Q^{\varepsilon, M}$ is actually concentrated on $C$. We shall now show that also $Q^{M}$ is concentrated on $C$ and that the family $\left\{Q^{M}: M \geqq\left|x_{0}\right|\right\}$ is tight in $C$. We shall then be able to take limits as $M \rightarrow \infty$ without checking the uniqueness of $Q^{M}$. in $M$

By assumption VI and (3.39) there exist constants $A$ and $B$ such that uniformly

$$
\begin{array}{r}
\frac{1}{|\theta|^{2}} \sum_{i, j} \theta_{i} A_{i j}^{M}(x) \theta_{j} \leqq A \\
\sum_{j}\left(b_{j}^{M}(x)\right)^{2} \leqq B^{2} .
\end{array}
$$

By [11], Eq. (2.1) [note that the proof of this estimate remains valid if $x^{M}(t)$ is merely in $D$ rather than $C]$ for each fixed $s \geqq 0, \Delta>0, \lambda>0$

$$
\begin{aligned}
& Q^{M}\left\{\sup _{s \leqq t \leqq s+\Delta}\left|X(t)-X(s)-\int_{s}^{t} b^{M}(X(\sigma)) d \sigma\right|>\lambda\right\} \\
& \leqq 2 d \exp -\frac{\lambda^{2}}{2 A d^{1 / 2} \Delta},
\end{aligned}
$$

and consequently for any $T \geqq 0$ and $\lambda>0$

$$
Q^{M}\left\{\sup _{\substack{0 \leqq s \leqq t \leqq T \\|\bar{t}-s| \leqq \Delta}}|X(t)-X(s)| \geqq 2 \lambda\right\} \leqq\left(\frac{T}{\Delta}+1\right) \exp -\frac{(\lambda-\Delta B)^{2}}{2 A d^{1 / 2} \Delta} \rightarrow 0
$$

as $\Delta \downarrow 0$, uniformly in $M$. Since also

$$
Q^{M}\left\{X(0)=x_{0}\right\}=\lim _{\varepsilon \downarrow 0} Q^{\varepsilon, M}\left\{x^{\varepsilon, M}(0)=x_{0}\right\}=1
$$

it follows from Theorem 8.2 in [5], that each $Q^{M}$ is concentrated on $C$ and that the family $\left\{Q^{M}\right\}_{M \geqq\left|x_{0}\right|}$ is tight on $C$. Moreover, since each $Q^{\varepsilon, M}$ is concentrated on $C, Q^{\varepsilon_{n}, M}$ converges weakly on $C$ to $Q^{M}$ as $n \rightarrow \infty$ (cf. [5], p. 151).

We can now easily complete the proof. Let $M_{k} \uparrow \infty$ be any sequence for which $Q^{M_{k}}$ converges weakly in $C$ to some measure $Q^{*}$ on $C$. By the tightness of $\left\{Q^{M}\right\}$ 
any sequence of $M$ 's tending to infinity contains a subsequence with this property. From the fact that the coefficients of $L^{M}$ converge boundedly, and uniformly on compacta, to those of $L$, and (3.42), it follows that

$$
f(X(t))-\int_{0}^{t} L F(X(\sigma)) d \sigma
$$

is a $\left(Q^{*}, \mathscr{M}_{0}^{t *}\right)$ martingale, where $\mathscr{M}_{u}^{v *}$ is the trace of $\mathscr{M}_{u}^{v *}$ on $C$. Of course [cf. (3.56)]

$$
Q^{*}\left\{X(0)=x_{0}\right\}=1 \text {. }
$$

By the hypotheses made in VI on $a_{i, j}$ and $b_{j}$ and by Theorems 6.2 and 5.6 of [12], respectively Theorem 2.3 and Remark 2.1 of [13], there is only one measure on $C$ with these properties, namely the one denoted by $R$ in Theorem 3. Thus $Q^{*}=R$ and

$$
Q^{M} \Rightarrow R \text { on } C .
$$

Now define for $X \in C$

$$
\tau(X, K)=\inf \{t \geqq 0:|X(t)|>K\} .
$$

We already observed in Step (ii) that $x^{\varepsilon, M}(t)=x^{\varepsilon}(t)$ up till the first time that $\left|x^{\varepsilon}(t)\right|=\frac{M}{2}$ so that for each $K \leqq \frac{M}{2}$

$$
\tau\left(x^{\varepsilon,} K\right)=\tau\left(x^{\varepsilon, M}, K\right) .
$$

Now let $S_{0}$ be a closed subset of $C\left([0, T] ; \mathbb{R}^{d}\right)$ and

$$
S=\left\{X \in C: X_{\mid[0, T]} \in S_{0}\right\} \text {. }
$$

Assume that we can show for each such $S$

$$
\limsup _{\varepsilon \downarrow 0} R^{\varepsilon}\{S\} \leqq R\{S\}
$$

It then follows from Theorem 2.1 in [5] that

$$
\lim _{\varepsilon \downarrow 0} R^{\varepsilon}\{\Psi\}=R\{\Psi\}
$$

for each bounded continuous functional $\Psi$ on $C$ with the property that $\Psi(X)$ depends on $X(\cdot)$ restricted to $[0, T]$ only. (Here we have written $R\{\Psi\}$ for the integral of $\Psi$ over $C$ with respect to the measure $R$.) As in [6] this result for every $T$ implies $R^{\varepsilon} \Rightarrow R$ on $C$. It therefore suffices to prove (3.60) for all $S$ of the form (3.59).

Let $\varepsilon_{n} \downarrow 0$. If necessary by going over to a subsequence we may assume that for each $M=1,2, \ldots$

$$
Q^{\varepsilon_{n}, M} \Rightarrow Q^{M} \quad \text { on } \quad C
$$

1 See also Theorem 6 in [19] 
for some probability measure $Q^{M}$ on $C$. It follows from (3.58) that for every $S$ of the form (3.59) and $K \leqq \frac{M}{2}$

$$
\begin{aligned}
R^{\varepsilon}\{\tau(X, K) \leqq T\} & =Q^{\varepsilon, M}\{\tau(X, K) \leqq T\} \\
R^{\varepsilon}\{S, \tau(X, K)>T\} & =Q^{\varepsilon, M}\{S, \tau(X, K)>T\}
\end{aligned}
$$

Consequently

$$
\begin{aligned}
\limsup _{n \rightarrow \infty} R^{\varepsilon_{n}}(S) & \leqq \limsup _{n \rightarrow \infty} Q^{\varepsilon_{n}, M}\{S\}+\limsup _{n \rightarrow \infty} Q^{\varepsilon_{n}, M}\{\tau(X, K) \leqq T\} \\
& \leqq Q^{M}\{S\}+Q^{M}\left\{\sup _{0 \leqq t \leqq T}|X(t)| \geqq K-1\right\} \quad[\text { by (3.62)]. }
\end{aligned}
$$

If we let $M \rightarrow \infty$ we obtain from this and (3.57)

$$
\limsup _{n \rightarrow \infty} R^{\varepsilon_{n}}\{S\} \leqq R\{S\}+R\left\{\sup _{0 \leqq t \leqq T}|X(t)| \geqq K-2\right\},
$$

and finally as $K \rightarrow \infty$ we obtain (3.60). This completes the proof of Theorem 3 .

\section{Appendix, Examples}

a) Gaussian Random Fields. Let $F: \mathbb{R}^{d} \times \Omega \rightarrow \mathbb{R}^{d}$ be a stationary (i.e. with distribution invariant under translations; cf. Sect. 2) Gaussian random field with mean zero and correlation matrix

$$
r_{k l}(y)=E\left\{F_{k}(x) F_{l}(x+y)\right\}, \quad x, y \in \mathbb{R}^{d}, \quad 1 \leqq k, l \leqq d .
$$

By Bochner's theorem ([14], Chap. 1.5) there exist totally finite complex valued measures $\mu_{k, l}$ on $\mathbb{R}^{d}$ such that

$$
r_{k l}(y)=\int_{\mathbb{R}^{d}} e^{i(\lambda, y)} \mu_{k, l}(d \lambda),
$$

and for each Borel set $B$ the matrix

$$
\left(\int_{B} \mu_{k, l}(d \lambda)\right)_{1 \leqq k, l \leqq d} \text { is hermitian and nonnegative definite. }
$$

$\mu_{k, l}$ is the so called spectral measure. We shall assume that the $\mu_{k, l}$ have densities, i.e. for some $L^{1}\left(\mathbb{R}^{d}\right)$ functions $f_{k, l}$

$$
r_{k l}(y)=\int_{\mathbb{R}^{d}} e^{i(\lambda, y)} f_{k, l}(\lambda) d \lambda
$$

Without loss of generality we may assume that for each $\lambda$ the matrix

$$
\left(f_{k, l}(\lambda)\right)_{1 \leqq k, l \leqq d} \text { is hermitian and nonnegative definite. }
$$

The $f_{k, l}$ take the place of the usual power spectral density. We define

$$
\tau(\lambda)=\text { smallest eigenvalue of }\left(f_{k, l}(\lambda)\right) \text {. }
$$


For simplicity we shall take the vector $v$ in (1.2) in the direction of the positive first coordinate axis, i.e.

$$
v=(|v|, 0, \ldots, 0) \text {. }
$$

We shall write $\lambda \in \mathbb{R}^{d}$ as $\left(\lambda_{1}, \lambda_{2}\right)$ with $\lambda_{1} \in \mathbb{R}, \lambda_{2} \in \mathbb{R}^{d-1}$. We then have the following

Theorem 4. Let $F$ be a stationary zero mean Gaussian random field as above. Assume that $f_{k, l}$ as in (4.4) exist and that for some $\alpha>1$

$$
\int_{\lambda \in \mathbb{R}^{d},|\lambda| \geqq 1}|\lambda|^{6}(\log \lambda)^{\alpha} f_{k, k}(\lambda) d \lambda<\infty, \quad 1 \leqq k \leqq d .
$$

If in addition (4.7) holds and for some integers $p=p(k, l) \geqq 7+2 d$ and constants $r \geqq 0$ and $A<\infty$

$$
\begin{aligned}
& \left|\left(\frac{\partial}{\partial \lambda_{1}}\right)^{p} f_{k, l}\left(\lambda_{1}+t, \lambda_{2}\right)\right| \leqq A(1+t)^{r} \tau\left(\lambda_{1}, \lambda_{2}\right) \\
& \text { for all } \lambda \in \mathbb{R}^{d}, t \in \mathbb{R} \text { and } 1 \leqq k, l \leqq d,
\end{aligned}
$$

then $F$ satisfies the conditions of Theorem 1 (even with $M=\infty$ in (2.7)).

Remark 4. (4.8) could be sharpened slightly by a more carefull application of Theorem 2.10 of [15]. If $\left(f_{k, l}(\lambda)\right)$ is nonsingular for all $\lambda$ and $p(k, l)$ is independent of $k, l$ it is also possible to replace (4.9) by the slightly less restrictive condition

$$
\begin{aligned}
& \sup _{\substack{\lambda \in \mathbb{R}^{d} \\
t \in \mathbb{R}^{d}}}(1+t)^{-r} \\
& \quad \cdot\left\{\text { largest eigenvalue of }\left(\frac{\partial}{\partial \lambda_{1}}\right)^{p} f\left(\lambda_{1}+t, \lambda_{2}\right) f^{-1}\left(\lambda_{1}, \lambda_{2}\right)\right\} \leqq A .
\end{aligned}
$$

Here $f^{-1}$ stands for the inverse matrix of $\left(f_{k, l}\right)$ and $\left(\frac{\partial}{\partial \lambda_{1}}\right)^{p} f$ is the matrix $\left(\left(\frac{\partial}{\partial \lambda_{1}}\right)^{p} f_{k, l}\right)$. Neither of these refinements seem useful for finding actual examples. It is helpful, though, to observe that one may replace $\tau$ in (4.9) by its lower bound (see [16], Sect. 5)

$$
\min _{k}\left\{f_{k, k}\left(\lambda_{1}, \lambda_{2}\right)-\sum_{l \neq k}\left|f_{k, l}\left(\lambda_{1}, \lambda_{2}\right)\right|\right\}^{+} .
$$

Example. Set

$$
R^{2}=\alpha_{1}\left(\lambda_{1}-\beta_{1}\right)^{2}+\sum_{i=2}^{d-1} \alpha_{i}\left(\lambda_{2, i}-\beta_{i}\right)^{2}
$$

for some $\alpha_{i}>0, \beta_{i} \in \mathbb{R}$ and $\lambda_{2}=\left(\lambda_{2,2}, \ldots, \lambda_{2, d}\right)$. Assume that

$$
f_{k, l}\left(\lambda_{1}, \lambda_{2}\right)=\frac{P_{k, l}\left(R^{2}\right)}{Q_{k, l}\left(R^{2}\right)}
$$


where $P_{k, l}(\cdot)$ and $Q_{k, l}(\cdot)$ are polynomials. Assume that $\left(f_{k, l}\left(\lambda_{1}, \lambda_{2}\right)\right)$ is positive definite for all $\lambda$ and that in addition for all $k, l$ and some constants $x_{0} \geqq 0,0<\gamma<1$,

$$
\begin{aligned}
& \inf _{x}\left|Q_{k, l}(x)\right|>0 \quad \text { and for all } x P_{k, k}(x)>0, Q_{k, k}(x)>0 ; \\
& \sum_{j \neq k}\left|\frac{P_{k, j}(x)}{Q_{k, j}(x)}\right| \leqq \gamma \frac{P_{k, k}(x)}{Q_{k, k}(x)} \text { for all }|x| \geqq x_{0} ; \\
& \text { degree } Q_{k, k}>\text { degree } P_{k, k}+\frac{1}{2}(d+6) .
\end{aligned}
$$

Then a Gaussian field with spectral density $\left(f_{k, l}\right)$ satisfies the conditions of Theorem 1.

Proof. It is almost immediate from (4.8) that $s_{i}(x)=-\left(\frac{\partial}{\partial x_{i}}\right)^{2} r_{k k}(x)$ exists, is finite and satisfies

$$
\left|s_{i}(0)-s_{i}(x)\right| \leqq C\left(\log \frac{1}{|x|}\right)^{-\alpha}
$$

for some constant $C$ and $|x| \leqq 1$. It follows from Theorem 2.10 in [15] that $s_{i}(\cdot)$ is the correlation function of some continuous and stationary Gaussian process, $Y_{k, i}(y, \omega)$ say. One can further take $X_{k}\left(x_{1}, \ldots, x_{i-1}, 0, \ldots, x_{d}\right)$ Gaussian and such that

$$
Z_{k}(x) \equiv X_{k}\left(x_{1}, \ldots, x_{i-1}, 0, \ldots, x_{d}\right)+\int_{0}^{x_{2}} Y_{k, i}\left(x_{1}, \ldots, x_{i-1}, t, \ldots, x_{d}\right) d t
$$

has the correlation function $r_{k k}(\cdot)$ and therefore has the same distribution function as $F_{k}(x)$. From this it follows that any continuous version of $F_{k}$ has almost surely a continuous partial derivative w.r.t. $x_{i}$ which has the same distribution as $Y_{k, i}$. Repeating this argument we see that almost surely $F_{k} \in C^{3}\left(\mathbb{R}^{d}\right)$. Since the derivatives of order $\leqq 3$ of $F_{k}$ are again mean zero continuous stationary Gaussian processes (2.2) is automatic (cf. [17], Theorem 8). It remains to verify (2.7). By a result of Kolmogorov and Rozanov ([14], Theorems IV.10.1 and IV.10.2) and (4.7) we have for all $M$, including $M=\infty$,

$$
\alpha(t, v, M) \leqq \varrho(t),
$$

where the "maximal correlation coefficient" $\varrho$ is given by

$$
\varrho(t)=\sup (E\{Y Z\}-E\{Y\} E\{Z\})
$$

with the sup taken over all random variables $Y, Z$ with $\sigma^{2}(Y)=\sigma^{2}(Z)=1$, and $Y$ in the linear span of $\{F(x, \cdot):(x, v) \leqq 0\}$ and $Z$ in the linear span of $\left\{F(x, \cdot): \frac{(x, v)}{|v|} \geqq t\right\}$. Therefore Theorem 4 is implied by the Proposition below, which is modeled after [14], Lemma IV.10.6.

Proposition. If $F$ is a stationary zero mean Gaussian random field whose spectral density $\left(f_{k, l}\right)$ satisfies (4.9), then

$$
\varrho(t) \leqq d C A t^{-q},
$$

where $q=\min _{k, l} p(k, l)$ and $C$ depends on $r$ and the $p(k, l)$ only. 
Remark 5. We can use the argument of [18], Lemma VI.1.1 to obtain further examples of random fields which have the required mixing properties. Indeed if $\tilde{f}_{k, l}=\sum_{i, j} \Gamma_{k, i} f_{k, l} \bar{\Gamma}_{l, j}$, where the $\Gamma_{k, i}$ are entire functions whose Fourier transform has support in $[-T,+T]$, then the maximal correlation coefficient $\tilde{\varrho}$ corresponding to $\tilde{f}$ satisfies

$$
\tilde{\varrho}(t) \leqq \varrho(t-2 T) .
$$

Proof. Standard manipulations show that

$$
\varrho(t)=\sup \left|\int_{\mathbb{R}^{d}}\left\{\sum_{1 \leqq k, l \leqq d} \varphi_{k}(\lambda) \psi_{l}(\lambda) e^{i t \lambda_{1}} f_{k, l}(\lambda)\right\} d \lambda\right|,
$$

where the sup is over all complex valued vectors of functions $\varphi_{k}(\lambda), \psi_{l}(\lambda)$ of unit norm in the space $H_{f}^{2}$, which is the closure of all functions of the form

$$
\sum_{v} \gamma_{v} \exp i\left(\lambda, x_{v}\right) \quad \text { (a finite sum) }
$$

with the first coordinate of $x_{v} \geqq 0$, in the norm

$$
\left\|\varphi_{k}\right\|_{f}^{2}=\int_{\mathbb{R}^{d}}\left\{\sum_{k, l} \varphi_{k}(\lambda) f_{k, l}(\lambda) \bar{\varphi}_{l}(\lambda)\right\} d \lambda .
$$

Now take

$$
2 m \geqq r+\max _{k, l} p(k, l)+2
$$

and

$$
Q_{k, l}\left(\lambda_{1}, \lambda_{2}\right)=Q_{k, l}\left(\lambda_{1}, \lambda_{2} ; \sigma\right) \equiv \frac{1}{\gamma_{m}} \int_{-\infty}^{+\infty} g_{\sigma, m}(x) \sum_{v=1}^{p}(-1)^{v-1}\left(\begin{array}{l}
p \\
v
\end{array}\right) f_{k, l}\left(\lambda_{1}+v x, \lambda_{2}\right) d x,
$$

where $p=p(k, l)$,

$$
g_{\sigma, m}(x)=\sigma\left(\frac{\sin \sigma x}{\sigma x}\right)^{2 m}=\sigma g_{1, m}(\sigma x)
$$

and

$$
\gamma_{m}=\int_{-\infty}^{+\infty} g_{1, m}(x) d x .
$$

We note that for all $\lambda_{2}$ with

$$
\int_{-\infty}^{+\infty}\left|f_{k, l}\left(\lambda_{1}, \lambda_{2}\right)\right| d \lambda_{1}<\infty \text { for all } 1 \leqq k, l \leqq d
$$

(i.e., for almost all $\lambda_{2}$ ) we have

$$
\int g_{\sigma, m}(x)\left|f_{k, l}\left(\lambda_{1}+v x, \lambda_{2}\right)\right| d x<\infty .
$$


This follows from the boundedness of $g_{\sigma, m}$. The principal estimate is that for all $\lambda_{2}$ satisfying (4.24) and all $\lambda_{1}$ and $\sigma \geqq 1$

$$
\left|f_{k, l}\left(\lambda_{1}, \lambda_{2}\right)-Q_{k, l}\left(\lambda_{1}, \lambda_{2}\right)\right| \leqq C A \sigma^{-p(k, l)} \tau\left(\lambda_{1}, \lambda_{2}\right)
$$

To prove (4.25) observe that its left hand side is bounded above by

$$
\frac{1}{\gamma_{m}} \int_{-\infty}^{+\infty} g_{\sigma, m}(x)\left|\sum_{\nu=0}^{p}(-1)^{v-1}\left(\begin{array}{l}
p \\
v
\end{array}\right) f_{k, l}\left(\lambda_{1}+v x, \lambda_{2}\right)\right| d x
$$

[note that (4.26) differs from (4.23) by a term with $v=0$ ]. Now all $x$ derivatives at $x=0$ up to order $p-1$ of

$$
\sum_{v=0}^{p}(-1)^{v-1}\left(\begin{array}{l}
p \\
v
\end{array}\right) f_{k, l}\left(\lambda_{1}+v x, \lambda_{2}\right)
$$

vanish, so that its absolute value is at most

$$
\begin{aligned}
& C_{p}|x|^{p} \sup _{|t| \leqq p|x|}\left|\left(\frac{\partial}{\partial \lambda_{1}}\right)^{p} f_{k, l}\left(\lambda_{1}+t, \lambda_{2}\right)\right| \\
& \leqq C_{p} A|x|^{p}(1+p|x|)^{r} \tau\left(\lambda_{1}, \lambda_{2}\right) \quad[\text { by (4.9)]. }
\end{aligned}
$$

Substitution of (4.27) into (4.26) gives

$$
\begin{aligned}
& \left|f_{k, l}\left(\lambda_{1}, \lambda_{2}\right)-Q_{k, l}\left(\lambda_{1} \lambda_{2}\right)\right| \\
& \quad \leqq C_{p} A \tau\left(\lambda_{1} \lambda_{2}\right) \frac{1}{\gamma_{m}} \int_{-\infty}^{+\infty} g_{\sigma, m}(x)|x|^{p}(1+p|x|)^{r} d x \\
& \quad \leqq 2^{r} C_{p} A \tau\left(\lambda_{1}, \lambda_{2}\right) \frac{1}{\gamma_{m}} \int_{-\infty}^{+\infty} g_{\sigma, m}\left\{\frac{|\sigma x|^{p}}{\sigma^{p}}+p^{r} \frac{|\sigma x|^{p+r}}{\sigma^{p+r}}\right\} d x \\
& \quad \leqq A \tau\left(\lambda_{1}, \lambda_{2}\right)\left\{\Gamma_{p, m, 0} \sigma^{-p}+\Gamma_{p, m, r} \sigma^{-p-r}\right\},
\end{aligned}
$$

where [see (4.22)]

$$
\begin{aligned}
\Gamma_{p . m, s} & =p^{s} 2^{r} C_{p} \frac{1}{\gamma_{m}} \int_{-\infty}^{+\infty} g_{\sigma, m}(x)|\sigma x|^{p+s} d x \\
& =r^{s} 2^{r} C_{p} \frac{1}{\gamma_{m}} \int_{-\infty}^{+\infty}(\sin x)^{2 m} x^{p+s-2 m} d x<\infty .
\end{aligned}
$$

This proves (4.25).

By the minimax characterization of eigenvalues

$$
\sum_{k, l} \varphi_{k}(\lambda) f_{k, l}(\lambda) \bar{\varphi}_{l}(\lambda) \geqq \tau\left(\lambda_{1} \lambda_{2}\right) \sum_{k}\left|\varphi_{k}(\lambda)\right|^{2}
$$

It follows easily from (4.28) and (4.25) that for all $\sigma \geqq 1$ and $\|\varphi\|_{f},\|\psi\|_{f}$ finite

$$
\int_{\mathbb{R}_{i}^{d}}\left|\sum_{h, l} \varphi_{k}(\lambda) \psi_{l}(\lambda) e^{i t \lambda_{1}} Q_{k, l}\left(\lambda_{1}, \lambda_{2}\right)\right| d \lambda<\infty .
$$


We claim that for $\sigma<t$

$$
\int\left\{\sum_{k, l} \varphi_{k}(\lambda) \psi_{l}(\lambda) e^{i t \lambda_{1}} Q_{k, l}\left(\lambda_{1}, \lambda_{2}\right)\right\} d \lambda=0
$$

To see this, let

$$
\begin{aligned}
& \varphi_{k}^{n}(\lambda)=\sum_{v} \gamma_{v, k}^{n} \exp i\left(\lambda, x_{k, v}^{n}\right), \\
& \psi_{l}^{n}(\lambda)=\sum_{v} \delta_{v, l}^{n} \exp i\left(\lambda, y_{k, v}^{n}\right),
\end{aligned}
$$

with the first coordinates of $x_{k, v}^{n}$ and $y_{k, v}^{n} \geqq 0$, be such that $\left(\varphi_{k}^{n}\right) \rightarrow\left(\varphi_{k}\right),\left(\psi_{l}^{n}\right) \rightarrow\left(\psi_{l}\right)$ in $H_{f}^{2}$ as $n \rightarrow \infty$. By virtue of the above estimates (4.30) is the limit of

$$
\int_{\mathbb{R}^{d}}\left\{\sum_{k, l} \varphi_{k}^{n}(\lambda) \psi_{l}^{n}(\lambda) e^{i t \lambda_{1}} Q_{k, l}(\lambda)\right\} d \lambda
$$

as $n \rightarrow \infty$. Thus it suffices to prove

$$
\int_{\mathbb{R}^{d}} e^{i s \lambda_{1}} Q_{k, l}\left(\lambda_{1}, \lambda_{2}\right) d \lambda_{1}=0
$$

whenever $s \geqq t>\sigma$ and (4.24) holds. This, however, is easy because for fixed $\lambda_{2}, Q_{k, l}\left(\lambda_{1}, \lambda_{2}\right)$ is a convolution of translates of $f$ and $g_{\sigma, m}$, and the Fourier transform of $g_{\sigma, m}$ has support in $[-\sigma,+\sigma]$.

The proof of (4.19) is now easily completed. For $\varphi, \psi \in H_{f}^{2}$ with norm equal to 1 we have for $1 \leqq \sigma<t$

$$
\begin{aligned}
&\left|\int_{\mathbb{R}^{d}}\left\{\sum_{k, l} \varphi_{k}(\lambda) \psi_{l}(\lambda) e^{i t \lambda_{1}} f_{k, l}(\lambda)\right\} d \lambda\right| \\
&=\left|\int_{\mathbb{R}^{d}}\left\{\sum_{k, l} \varphi_{k}(\lambda) \psi_{l}(\lambda) e^{i t \lambda_{1}}\left[f_{k, l}(\lambda)-Q_{k, l}(\lambda)\right]\right\} d \lambda\right| \\
& \leqq C A \sigma^{-q} \sum_{k, l} \int\left|\varphi_{k}(\lambda) \| \psi_{l}(\lambda)\right| \tau\left(\lambda_{1}, \lambda_{2}\right) d \lambda \\
& \leqq d C A \sigma^{-q} \int\left\{\sum_{k, l} \varphi_{k}(\lambda) f_{k, l}(\lambda) \bar{\varphi}_{l}(\lambda)\right\}^{1 / 2} \\
& \cdot\left\{\sum_{k, l} \psi_{k}(\lambda) f_{k, l}(\lambda) \bar{\psi}_{l}(\lambda)\right\}^{1 / 2} d \lambda
\end{aligned}
$$

[by Schwarz' inequality and (4.28)]. A final application of Schwarz' inequality shows that (4.32) is bounded by

$$
d C A \sigma^{-q}\|\varphi\|_{f}\|\psi\|_{f}=d C A \sigma^{-q} .
$$

b) Trivial Gaussian examples for $F$ satisfying the conditions of Theorem 1 can be obtained by taking $F$ a stationary mean zero Gaussian random field with a correlation matrix $r_{k l}(y)$ which is seven times continuously differentiable [this implies $F \in C^{3}$ and (2.2) as in a)] and which vanishes for $\left|y_{1}\right| \geqq t_{0}$. [In the latter case $\varrho(t)$, and hence $\alpha(t, v, M)$ vanish for $t>t_{0}$.] Any matrix of the form

$$
r_{k l}(y)=\int_{\mathbb{R}^{d}} \sum_{m} s_{k, m}(x) s_{l, m}(x+y) d x
$$


with $\left(s_{k, m}(x)\right)$ a rectangular matrix of real valued

$$
s_{k, m}(\cdot) \in C^{7}\left(\mathbb{R}^{d}\right) \cap L^{1}\left(\mathbb{R}^{d}\right), \quad s_{k, m}(x)=0 \quad \text { for } \quad\left|x_{1}\right| \geqq \frac{t_{0}}{2}
$$

can serve as such a correlation matrix. Indeed (4.33) is easily seen to be nonnegative definite.

c) Poisson Blobs. Let $F$ be any random field (not necessarily Gaussian) which satisfies the conditions of Theorem 1 with $M=\infty$ in (2.7). (E.g. $F \equiv 0$ is permitted.) It is then possible to obtain a new field, $\tilde{F}$ say, which still satisfies the hypotheses of Theorem 1 by "perturbing $F$ on the union of a countable number of randomly located balls". More precisely, let $P_{\varrho}$ be a Poisson point process on $[0, \infty) \times \mathbb{R}^{d}$ with intensity $\varrho G(d r) \times d \lambda$, where $G$ is some probability distribution on $[0, \infty)$ and $\lambda$ is Lebesgue measure on $\mathbb{R}^{d}$. I.e., $N(B) \equiv$ the number of points $\left(r_{i}, p_{i}\right)$ in the Borel set $B \subset[0, \infty) \times \mathbb{R}^{d}$ has a Poisson distribution with mean $\varrho G \times \lambda(B) ;$ if $B_{1}, \ldots, B_{k}$ are disjoint then $N\left(B_{1}\right), \ldots, N\left(B_{k}\right)$ are independent. In addition let $Z_{1}, Z_{2}, \ldots$ be a sequence of independent identically distributed random variables. We shall assume that

$$
P_{\varrho}, Z_{1}, Z_{2}, \ldots, \quad \text { and the field } F \text { are independent of each other. }
$$

For the $j^{\text {th }}$ point (in some ordering) $\left(r_{j}, p_{j}\right)$ of $P_{\varrho}$ we form the ball

$$
B_{j}=\left\{x \in \mathbb{R}^{d}:\left|x-p_{j}\right| \leqq r_{j}\right\} .
$$

In addition we introduce

$$
\Delta=\bigcup_{j} B_{j}
$$

and for $x \in \mathbb{R}^{d}$, the union of those $B_{i}$ which contain $x$, i.e.,

$$
D(x)=\bigcup_{B_{j} \ni x} B_{j} .
$$

We do not change $F$ on the complement of $\Delta$, i.e. $\tilde{F}(x, \omega)=F(x, \omega)$ for $x \notin \Delta$. For $x \in \Delta$ we perturb $F$ in any way which depends only on $D(x)$, the values of $F$ on $D(x)$ and those $Z_{j}$ for which $B_{j}$ contains $x$. Roughly speaking for each ball $B_{j}$ which contains $x$ there is some effect on $F(x)$ and this effect may still have some random element in it, because it may depend on the extraneous random variable $Z_{j}$. For instance the perturbations might be additive

$$
\tilde{F}(x)=F(x)+\sum_{B_{j} \ni x} G_{j}\left(x, Z_{j}\right),
$$

where $G_{j}\left(\cdot, Z_{j}\right)$ is some $C^{3}\left(\mathbb{R}^{d}\right)$ function with support in $B_{j}$. Of course we must make sure that $\tilde{F}$ is still jointly measurable, stationary, belongs to $C^{3}\left(\mathbb{R}^{d}\right)$ and satisfies (2.2) and (2.3). This, however, is easy to achieve and here we only give a condition which guarantees that $\tilde{F}$ again satisfies (2.7).

Theorem 5. Let $F$ and $\tilde{F}$ be as above and $\alpha(t, v, M)$ be defined by (2.4)-(2.6) and similarly for $\tilde{\alpha}(t, v, M)$ with $F$ replaced by $\tilde{F}$. If

$$
\int_{0}^{\infty}\{\alpha(t, v, \infty)\}^{1 / p} d t<\infty
$$


and

$$
\int_{0}^{\infty} d t\left\{\int_{t}^{\infty} w^{d-1} G([w, \infty)) d w\right\}^{1 / p}<\infty,
$$

then for every $M<\infty$ also

$$
\int_{0}^{\infty}\{\tilde{\alpha}(t, v, M)\}^{1 / p} d t<\infty .
$$

We shall merely give a heuristic argument for this theorem. Consider the event $E_{1}(t)$ defined as follows:

All the balls $B_{j}$ which intersect the strip

$$
S_{1}=\left\{x \in \mathbb{R}^{d}:(x, v) \leqq 0,\left|x-\frac{(x, v)}{|v|^{2}} v\right| \leqq M\right\}
$$

lie entirely in the half space

$$
H_{1}=\left\{x \in \mathbb{R}^{d}: \frac{(x, v)}{|v|} \leqq \frac{t}{4}\right\} .
$$

Similarly we define $E_{2}(t)$ to be the event that all balls $B_{j}$ which intersect the strip

$$
S_{2}=\left\{x \in \mathbb{R}^{d}: \frac{(x, v)}{|v|} \geqq t,\left|x-\frac{(x, v)}{|v|^{2}} v\right| \leqq M\right\}
$$

lie entirely in the half space

$$
H_{2}=\left\{x \in \mathbb{R}^{d}: \frac{(x, v)}{|v|} \geqq \frac{3 t}{4}\right\} .
$$

$E_{1}, E_{2}$ are independent of each other and depend on $P_{\varrho}$ only. If a realization of $P_{\varrho}$ is given, such that $E_{1}$ occurs, and if $Z_{1}, Z_{2}, \ldots$ are given, then $\left\{\tilde{F}(x): x \in S_{1}\right\}$ depends only on $\left\{F(y): y \in H_{1}\right\}$, and similarly if the subscript 1 is replaced by 2 . Since the $\sigma$-fields $\mathscr{F}_{-\infty}^{t / 4}(v, \infty)$ and $\mathscr{F}_{3 t / 4}^{\infty}(\mathrm{v}, \mathrm{M})$ [see $(2.4)$ for notation] are "independent up to an error $\alpha\left(\frac{t}{2}, v, \infty\right)$ " one can conclude that for any events $\tilde{A}_{1} \in \tilde{\mathscr{F}}_{-\infty}^{0}(v, M)$ [defined as in (2.4), with $\tilde{F}$ replacing $F]$ and $\tilde{B} \in \tilde{\mathscr{F}}_{t}^{\infty}(v, M)$ one has

$$
\left|P\left\{\tilde{A} \tilde{B} E_{1} E_{2}\right\}-P\left\{\tilde{A} E_{1}\right\} P\left\{\tilde{B} E_{2}\right\}\right| \leqq \alpha\left(\frac{t}{2}, v, \infty\right) .
$$

Consequently

$$
\begin{aligned}
\tilde{\alpha}(t, v, M) \leqq & \alpha\left(\frac{t}{2}, v, \infty\right)+2 P\left\{E_{1} \text { does not occur }\right\} \\
& +2 P\left\{E_{2} \text { does not occur }\right\} .
\end{aligned}
$$

It is routine to estimate $P\left\{E_{i}\right.$ does not occur $\}$ and to derive (4.37) from (4.38), (4.35), and (4.36). 


\section{References}

1. Monin, A.S., Yaglom, A.M.: Statistical fluid mechanics: mechanics of turbulence, Vol. 1. Cambridge: MIT Press 1971

2. Roberts, P.H.: Analytical theory of turbulent diffusion. J. Fluid Mech. 11, 257-283 (1961)

3. Kraichnan, R.H.: Diffusion by a random velocity field. Phys. Fluids 13, 22-31 (1970)

4. Kubo, R.: Stochastic Liouville equation. J. Math. Phys. 4, 174-183 (1963)

5. Billingsley, P.: Convergence of probability measures. New York: Wiley 1968

6. Stone, C.: Weak convergence of stochastic processes defined on semi-infinite time intervals. Proc. Am. Math. Soc. 14, 694-696 (1963)

7. Ibragimov, I.A., Linnik, Yu.V.: Independent and stationary sequences of random variables. Groningen: Wolters-Noordhoff 1971

8. Khasminskii, R.Z.: A limit theorem for solutions of differential equations with a random right hand side. Theor. Prob. Appl. 11, 390-406 (1966)

9. Papanicolaou, G.C., Kohler, W.: Asymptotic theory of mixing stochastic ordinary differential equations. Comm. Pure Appl. Math. 27, 641-668 (1974)

10. Borodin, A. N. : A limit theorem for solutions of differential equations with random right hand side. Teor. Veroyatn. Ee Primen 22, 498-512 (1977)

11. Stroock, D., Varadhan, S. R.S. : Diffusion processes with boundary conditions. Comm. Pure Appl. Math. 24, 147-225 (1971)

12. Stroock, D., Varadhan, S. R.S. : Diffusion processes with continuous coefficients. I., II. Comm. Pure Appl. Math. 22, 345-400, 479-530 (1969)

13. Stroock, D., Varadhan, S.R.S.: On the support of diffusion processes with applications to the strong maximum principle. Proc. 6th Berkeley Symp. Math. Statist. and Prob., Vol. III, pp. 333-359. Berkeley, California: University of California Press 1972

14. Rozanov, Yu.A.: Stationary random processes. San Francisco. California: Holden-Day 1967

15. Dudley, R.M. : Sample functions of the Gaussian process. Ann. Prob. 1, 66-103 (1973)

16. Taussky, O.: A recurring theorem on determinants. Am. Math. Monthly 56, 672-675 (1949)

17. Fernique, X.: Régularité de processus Gaussiens. Inventiones Math. 12, 304-320 (1971)

18. Ibragimov, I.A., Rozanov, Yu. A.: Processus aléatoires Gaussiens. Moscow: Editions MIR 1974

19. Whitt, W.: Weak convergence of probability measures on the function space $C[0, \infty)$. Ann. Math. Stat. 41, 939-944 (1970)

Communicated by J. L. Lebowitz

Received August 3, 1978 